\title{
Maternal Piwi Regulates Primordial Germ Cell Development to Ensure the Fertility of Female Progeny in Drosophila
}

\author{
Lauren E Gonzalez ${ }^{1,2}$, Xiongzhuo Tang ${ }^{1,3}$, Haifan Lin ${ }^{1,3,4}$ \\ ${ }^{1}$ Yale Stem Cell Center, Yale School of Medicine, New Haven, CT 06519, USA \\ ${ }^{2}$ Department of Genetics, Yale School of Medicine, New Haven, CT 06519, USA \\ ${ }^{3}$ Department of Cell Biology, Yale School of Medicine, New Haven, CT 06519, USA
}

Running Title: Maternal Piwi in F1 Female Fertility

Key words: Piwi, maternal effect, germline development, oogenesis, Drosophila

${ }^{4}$ Corresponding Author: Haifan Lin, Phone: 203-785-6239

Email: haifan.lin@yale.edu 


\begin{abstract}
In many animals, germline development is initiated by proteins and RNAs that are expressed maternally. PIWI proteins and their associated small noncoding PIWI-interacting RNAs (piRNAs), which guide PIWI to target RNAs by base-pairing, are among the maternal components deposited into the germline of the early embryo in Drosophila. Piwi has been extensively studied in the adult ovary and testis, where it is required for transposon suppression, germline stem cell self-renewal, and fertility. Consequently, loss of Piwi in the adult ovary using piwi-null alleles or knockdown from early oogenesis results in complete sterility, limiting investigation into possible embryonic functions of maternal Piwi. In this study, we show that the maternal Piwi protein persists in the embryonic germline through gonad coalescence, suggesting that maternal Piwi can regulate germline development beyond early embryogenesis. Using a maternal knockdown strategy, we find that maternal Piwi is required for the fertility and normal gonad morphology of female, but not male, progeny. Following maternal piwi knockdown, transposons were mildly derepressed in the early embryo but were fully repressed in the ovaries of adult progeny. Furthermore, the maternal piRNA pool was diminished, reducing the capacity of the PIWI/piRNA complex to target zygotic genes during embryogenesis. Examination of embryonic germ cell proliferation and ovarian gene expression showed that the germline of female progeny was partially masculinized by maternal piwi knockdown. Our study reveals a novel role for maternal Piwi in the germline development of female progeny and suggests that the PIWI/piRNA pathway is involved in germline sex determination in Drosophila.
\end{abstract}




\section{INTRODUCTION}

Maternally deposited proteins and mRNAs drive the earliest stages of embryonic development. This is achieved in a unique way during the germline development of many animals, including Drosophila. During Drosophila oogenesis, germline-determining maternal proteins and RNAs are enriched in the cytoplasm at the posterior of the developing oocyte, in a region called the germ plasm (Lehmann 2016). This concentration of germline determinants facilitates the rapid and local specification of posterior nuclei into primordial germ cells (PGCs) during the maternal phase of embryogenesis. PGCs remain largely transcriptionally quiescent for several hours after the zygotic transcriptome is activated in the soma (Siddiqui et al. 2012; Van Doren et al. 1998). Even after the activation of the zygotic genome in the germline, the migration of PGCs through the midgut and their coalescence into embryonic gonads is regulated by intrinsic maternal and zygotic factors, and by the surrounding somatic cells (Jemc 2011; Slaidina and Lehmann 2017; Wawersik et al. 2005).

Drosophila PIWI proteins - Piwi, Aubergine (Aub), and Argonaute3 (Ago3) - are among these germ plasm-enriched maternal factors (Brennecke et al. 2007; Harris and Macdonald 2001; Mani et al. 2014; Megosh et al. 2006). The vast majority of embryos laid by piwi-null, aubnull, and ago3-null females suffer chromosome segregation defects which result in embryonic arrest before gastrulation (Mani et al. 2014). Of the few embryos that progress beyond the first few cell cycles, those laid by aub-null and piwi-null females fail to specify PGCs (Harris and Macdonald 2001; Megosh et al. 2006). In the case of the embryos from aub-null females, this is likely because Aub is required for the enrichment of germ plasm mRNAs at the posterior of the embryo by stabilizing them in the posterior and destabilizing them in other regions (Barckmann et al. 2015; Rouget et al. 2010; Vourekas et al. 2016). Aub, like other PIWI proteins, can regulate such diverse RNAs because of their association with small noncoding PIWI-interacting RNAs (piRNAs) (Aravin et al. 2006; Brennecke et al. 2007; Girard et al. 2006; Grivna et al. 2006; Lau et al. 2006). Drosophila piRNAs are 23-30 nt long, extremely heterogeneous, and can 
bind target RNAs with imperfect complementarity (Gou et al. 2014; Halbach et al. 2020; Shen et al. 2018; Zhang et al. 2018). Thus, they can guide PIWI proteins to potentially regulate a large swath of the transcriptome (Barckmann et al. 2015; Lee et al. 2012; Vourekas et al. 2016). All three Drosophila PIWI proteins can target mRNAs in the cytoplasm and regulate them posttranscriptionally, but Piwi mainly targets RNAs in the nucleus and regulates them at the transcriptional level.

The PIWI/piRNA pathway has been mostly studied in adult gonads, where its bestknown role is in preventing transposon activation and protecting germ cells from transposoninduced genome instability (Wang and Lin 2021). Investigation into the role of PIWI/piRNA in transposon silencing was initially motivated by the observation that the majority of Drosophila piRNAs are complementary to transposons (Saito et al. 2006; Vagin et al. 2006). Building on this knowledge, it has been proposed that one major function of maternal PIWI/piRNA deposition into the germ plasm is to maintain transposon suppression across generations. This is exemplified in hybrid dysgenesis, in which progeny that paternally inherit a transposon but do not maternally inherit the corresponding transposon-targeting piRNAs cannot suppress the expression of that transposon (Akkouche et al. 2013; Brennecke et al. 2008). Consequently, hybrid dysgenesis typically results in genome instability, gonadal atrophy, and infertility (Dorogova et al. 2017; Engels and Preston 1979; Kidwell et al. 1977). Many studies argue that developmental defects in PIWI/piRNA mutants are caused by transposon derepression, but some studies suggest that transposon derepression is separable from other defects (Durdevic et al. 2018; Klenov et al. 2011).

Piwi and other PIWI/piRNA pathway proteins have been observed in germ cells throughout embryogenesis (Marie et al. 2017), and the function of zygotic Piwi in embryogenesis has begun to be explored in the context of transposon suppression (Akkouche et al. 2017; Marie et al. 2017). Although most Drosophila piRNAs target transposons, there are also piRNAs that target and regulate non-transposon genes in the early embryo (Barckmann et 
al. 2018; Barckmann et al. 2015; Dufourt et al. 2017; Ramat et al. 2020; Rouget et al. 2010), adult ovaries (Klein et al. 2016; Lin et al. 2020; Ma et al. 2017; Rojas-Rios et al. 2017), and adult testes (Gonzalez et al. 2015; Kotov et al. 2019; Nishida et al. 2007). This raises the possibility that maternal Piwi/piRNA could regulate non-transposon genes beyond early embryogenesis. To date, studies into this possibility have largely been limited by the severe cell cycle defects during early embryogenesis (Durdevic et al. 2018; Mani et al. 2014) and failure of PGC specification (Megosh et al. 2006) in progeny of females with mutations in PIWI/piRNA pathway genes. This challenge is further complicated in the case of Piwi itself, because piwi mutations block germline stem cell self-renewal, so piwi mutant females do not complete oogenesis (Cox et al. 1998; Cox et al. 2000). Only genetically mosaic females with Piwi expressed in ovarian somatic but not germline cells can lay eggs that lack maternal Piwi (Cox et al. 1998; Cox et al. 2000; Mani et al. 2014; Megosh et al. 2006). However, most of these eggs arrest in early embryogenesis (Cox et al. 1998; Mani et al. 2014). Because of these technical limitations, it remains unexplored whether maternal Piwi regulates any developmental processes beyond early embryonic stages.

In this study, we investigated the function of maternal Piwi on the germline development of progeny. We first observed that maternal Piwi persists in the embryonic germline at least through gonad coalescence. We then used a UAS/Gal4 approach to reduce but not completely eliminate maternal Piwi levels, which circumvents the aforementioned challenges of studying the function of maternal Piwi. Strikingly, we found that knockdown of maternal piwi decreased the fertility of female, but not male, progeny, suggesting that maternal Piwi plays a critical role in the germline development of female progeny. In contrast to other PIWI/piRNA depletion approaches, transposon expression was only mildly affected by maternal piwi knockdown. Transcriptome analysis, piRNA analysis, and examination of embryonic germ cell proliferation suggest that the germline of female progeny is partially masculinized by maternal piwi knockdown. These observations add to the growing body of evidence that the PIWI/piRNA 
pathway can regulate developmental processes independently of its transposon suppression function.

\section{METHODS}

\section{Fly strains and husbandry}

All Drosophila stocks were raised on standard agar/molasses medium and raised at $25^{\circ} \mathrm{C}$ for experiments. For all crosses involving UAS constructs and GAL4 constructs, females carried the UAS constructs and males carried the GAL4 constructs. Two anti-piwi shRNA lines were used: "Piwi RNAi \#1," which is BDSC stock \#37483 and targets piwi exon 3, and "Piwi RNAi \#2," which was generated in Dr. Ting Xie's lab (called “THU”) and targets piwi exon 2. $D f(2 L) E D 761$ and $z u c^{H M 27}$ fly stocks were gifts from Dr. Trudi Schupbach, and the Dfd-lacZ fly stock was a gift from Dr. Mark van Doren. See Reagent Table for more details about these and other fly stocks.

\section{Fertility tests}

For female fertility tests, virgin females were mated with two $w^{1118}$ males in individual vials for two days before beginning the fertility test. For each vial, the flies were transferred to a new vial every 24 hours, and the number of eggs in the old vial was counted within four hours of transferring the parents. If the female died during the fertility test, the day of death was recorded and fertility data were omitted. If a male died during the fertility test, it was replaced with another $w^{1118}$ male.

For male fertility tests, each vial contained one male with three virgin $w^{1118}$ females, vials were transferred every 24 hours, and the number of eggs in the old vial were counted within four hours of transferring the parents. On alternating days, the three females were replaced with three new virgin $w^{1118}$ females. If a female or male died during the fertility test, the day of death was recorded and fertility data were omitted. 
For all fertility tests: Minimal yeast was added to vials to minimize nutrition-related effects on fertility and to aid in visualization of eggs. Each vial was allowed to age until pupae began eclosing. Once pupae began eclosing, the number of adults were counted every day until all were eclosed. Percentage of adults eclosed was calculated as (\# total adults) / (\# embryos). Males and females were counted separately, and sex ratios were calculated as (total \# female adults) / (total \# male adults). Percentage of adults eclosed and sex ratios were only calculated for parents who laid at least 10 eggs. 18-22 total individuals of each genotype were tested across two experiments performed at different times.

\section{Immunostaining}

Ovaries from 2-3 day old females were dissected in 1× PBS, then fixed for 20 minutes in fixative $(0.5 \%(\mathrm{v} / \mathrm{v}) \mathrm{NP}-40,2 \%$ formaldehyde in PBS) at room temperature. Ovaries were then washed in PBST (0.1\% TritonX-100 in PBS) $3 \times$ for 15 minutes each, then blocked in 5\% normal goat serum in PBST (NGS) for one hour at room temperature or overnight at $4^{\circ} \mathrm{C}$. Blocking solution was replaced with primary antibody diluted in NGS, and samples incubated overnight at $4^{\circ} \mathrm{C}$. Ovaries were again washed at room temperature in PBST $3 \times$ for 15 minutes each, and incubated in secondary antibody diluted in NGS for four hours at room temperature or overnight at $4^{\circ} \mathrm{C}$. Ovaries were washed in PBST once, then incubated in DAPI diluted in PBST (1:5000) for 10 minutes and mounted in Vectashield.

Testes from 1-4 day old males were dissected in 1× PBS, then fixed in testis fixative (0.02\% Triton-X-100, 2\% formaldehyde in PBS) for 10 minutes, before being washed $3 \times$ for 15 minutes each in PBST (0.1\% Triton-X-100). From this point onwards, testicular immunostaining proceeded as for ovaries above.

The third instar larval gonads were dissected in $1 \times$ PBS and fixed in fixative (100 mM glutamic acid, 25 mM KCl, 20 mM MgSO4, 4 mM 4 mM Sodium Phosphate, 1mM MgCl2 and $4 \%$ paraformaldehyde) at room temperature for 40 min, then briefly washed in PBST (PBS with 
$0.1 \%$ Triton $\mathrm{X}-100)$ and blocked with blocking buffer (PBST+0.5\% normal goat serum) at room temperature for $30 \mathrm{~min}$, followed by overnight incubation with primary antibody at $4^{\circ} \mathrm{C}$. From this point onwards, immunostaining proceeded as described for ovaries above.

Embryos were collected on grape juice plates in embryo collection cages containing around 100 females and 20 males, washed in saline solution $(0.12 \mathrm{M} \mathrm{NaCl}, 0.03 \%$ Triton-X100), dechorionated in $50 \%$ bleach for two minutes, and washed thoroughly in deionized water. Embryos were then transferred to $50 \%$ heptane and $50 \%$ embryo fixation solution ( $1 \times$ PBS, 50 mM EGTA, 9.25\% formaldehyde) and incubated for 10-20 minutes, rocking at room temperature. After removing fixative, embryos were washed in ice-cold $100 \%$ methanol $(\mathrm{MeOH})$ and shaken for two minutes. Embryos were washed 3-4× in ice-cold $100 \% \mathrm{MeOH}$ and either stored in $\mathrm{MeOH}$ at $-20^{\circ} \mathrm{C}$ or immediately rehydrated in PBST by sequentially replacing $\mathrm{MeOH}$ with a MeOH:PBST series with increasing concentrations of PBST (5 minutes each in 70:30 MeOH:PBST, 50:50 MeOH:PBST, 30:70 MeOH:PBST, 100\% PBST). Embryos were blocked in NGS for one hour at room temperature or overnight at $4^{\circ} \mathrm{C}$, and immunostaining proceeded as described for ovaries above.

Samples were imaged using ZEISS Axio Imager2 or Leica TCS SP5 Spectral Confocal Microscope on sequential scanning mode, and image adjustments were made in ImageJ. See the Supplementary Reagent Table for details about antibodies and other commercial reagents.

\section{Western blotting}

0-1.5 h embryos were collected on grape juice plates in embryo collection cages containing about 100 females and 20 males. Embryos were washed with deionized water, dechorionated in $50 \%$ bleach for 1 minute, washed again thoroughly with water, and transferred to MCB buffer (50 mM Hepes/ $\mathrm{NaOH}$ (pH 7.5), $150 \mathrm{mM}$ potassium acetate, $2 \mathrm{mM}$ magnesium acetate, 10\% (v/v) glycerol, $1 \mathrm{mM}$ DTT, $0.1 \%(\mathrm{v} / \mathrm{v})$ Triton X-100, 0.1\% (v/v) NP-40, 1× protease inhibitor and $0.5 \%(\mathrm{v} / \mathrm{v})$ beta-mercaptoethanol). Sample was homogenized with a pestle, then 
centrifuged at $14000 \mathrm{~g}$ for 20 minutes $\left(4^{\circ} \mathrm{C}\right)$, and supernatant was transferred to a new tube. Approximately $50 \mu \mathrm{g}$ of protein sample, heat denatured with $6 \times$ SDS loading dye, was run on a $10 \%$ SDS-PAGE gel. Proteins were transferred to a PVDF membrane, incubated with the appropriate primary antibody at $4^{\circ} \mathrm{C}$ overnight, then detected with the appropriate HRP-labeled secondary antibody. See the Supplementary Reagent Table for details about antibodies and other commercial reagents.

\section{RNA extraction and RT-PCR}

Ovaries were extracted from 1-2 day-old females in 1× PBS, then transferred to TRIzol; RNA was extracted following manufacturer's instructions. 6-8 pairs of ovaries were grouped together for each sample. 0-1.5 h embryos were collected as described for Western Blotting above and transferred to $1 \times$ PBST. PBST was replaced with TRIzol; RNA was extracted following manufacturer's instructions.

Two $\mu \mathrm{g}$ of total RNA was used for reverse transcription using MultiScribe (ThermoFischer) following manufacturer's instructions, and diluted $5 \times$ before quantitative PCR. For quantitative PCR, transcripts of interest were amplified using iTaq Universal SYBR Green (BioRad) using manufacturer's recommended conditions, on a BioRad CFX96 Real-Time machine. All primer sets used for qPCR were confirmed to have amplification efficiency 80$110 \%$. Gene expression was calculated using the $\Delta \Delta \mathrm{C}_{\mathrm{T}}$ method, using act5C as the housekeeping gene and using GFP-MatKD as the control, unless otherwise specified. For nonquantitative PCR, genes of interest were amplified using GoTaq Green Master Mix (Promega) with the following reaction conditions: $95^{\circ} \mathrm{C} 120 \mathrm{~s}, 25 \times\left(95^{\circ} \mathrm{C} 30 \mathrm{~s}, 55^{\circ} \mathrm{C} 30 \mathrm{~s}, 72^{\circ} \mathrm{C} 60 \mathrm{~s}\right), 72^{\circ} \mathrm{C}$ 300s. See the Supplementary Reagent Table for details about primers and other commercial reagents. 


\section{Small RNA extraction, library preparation, and sequencing}

For Small RNA-Seq, $20 \mu \mathrm{g}$ of total RNA from 0-1.5 h embryos was run for 3.5 hours on a $15 \%$ polyacrylamide gel that had been pre-running in $1 \times$ TBE for at least one hour. RNA was visualized by incubating the gel in Diamond Dye (Promega) and the 20-29 nt area of the gel was cut out for purification. $20 \mathrm{nt} / 30 \mathrm{nt} / 70 \mathrm{nt}$ RNA ladder was custom made. RNA was purified by fragmenting the gel piece and incubating fragments in AES buffer (300 mM NaAc, 2 mM EDTA $0.1 \%(\mathrm{w} / \mathrm{v})$ SDS) overnight at room temperature. Supernatant was transferred to precipitation buffer (0.3 M NaAc, 90\% ethanol (EtOH), $1 \mu$ glycoblue (ThermoFischer) and incubated on ice for one hour. RNA was then centrifuged at $13000 \mathrm{~g}$ for 30 minutes, washed in $70 \% \mathrm{EtOH}$, resuspended in $10 \mu \mathrm{L}$ nuclease-free $\mathrm{H}_{2} \mathrm{O}$, and stored at $-80^{\circ} \mathrm{C} .5 \mu \mathrm{L}$ (approximately $75 \mathrm{ng} \mathrm{RNA}$ ) was used for library preparation.

Total RNA purified by TRIzol was used for library preparation for RNA-Seq. For both total RNA-Seq and Small RNA-Seq, three replicates of each sample were collected and analyzed. Sequencing libraries were prepared according to the Illumina TruSeq Small RNA Library Prep Kit or Illumina TruSeq Stranded mRNA Library prep protocols. All sequencing data were generated on a HiSeq2000 at the Yale Stem Cell Center Genomics Core and sequencing quality was assessed using FastQC (Andrews 2010).

\section{Bioinformatics analysis}

For Total RNA-Seq: reads were mapped to D. melanogaster Berkeley Drosophila Genome Project (BDGP) release 6 using STAR (Dobin et al. 2013), and counted at transposable element and gene features, defined by gene annotation in BDGP6.28, using TETools (Lerat et al. 2017). Expression level changes between experimental and control samples were calculated using DESeq2 (Anders and Huber 2010) with standard parameters.

For each RNAi line, genes which were differentially expressed $>1.5$-fold (compared to GFPMatKD) with a significance $\mathrm{p}$-adjusted $<0.05$ were considered to be differentially expressed. 
Gene Ontology analysis was performed as a PANTHER Overrepresentation Test using GO database released 2020-03-23 (doi: 10.5281/zenodo.3727280).

For Small RNA-Seq: Adapter sequence (TGGAATTCTCGGGTGCCAAGG) was trimmed from reads using CutAdapt. rRNA, miRNA, tRNA, and siRNA (according to FlyBase annotation, and including exogenous siRNAs being expressed by our RNAi lines:

TTGTAGTTGTACTCCAGCTTG for GFP RNAi, TTGATTTCGGAGTTTGTCCAA for Piwi RNAi \#1, and TTCGTCTGCAGCATGACCGGGG for Piwi RNAi \#2) were filtered out, and remaining sequences were then mapped to the Drosophila genome using Bowtie1.2.2 (Langmead et al. 2009) to create the list of putative piRNAs. Nucleotide composition was determined using FastQC (Andrews 2010). To identify putative piRNA targets on non-transposon mRNAs, these putative piRNAs were mapped using Bowtie1.2.2 (Langmead et al. 2009) to the transcribed sequences (BDGP sequence release 6, annotation release 25; dmel-all-gene-r6.26.fasta retrieved from FlyBase) of all $D$. melanogastar genes, excluding transposons. Mapping required the piRNA read to be antisense to the mRNA and allowed up to 2 mismatches in the first 24 nucleotides. Featurecounts (Liao et al. 2014) was used to count the number of piRNA reads that aligned to each mRNA, weighted for the number of mapping locations for each read. This count is referred to as "piRNA targetability." Targetability level changes between experimental and control samples was calculated using DESeq2 (Anders and Huber 2010) with standard parameters. For each RNAi line, genes which were differentially targetable $>1.5$-fold (compared to GFP-MatKD) with a significance $\mathrm{p}$-adjusted $<0.05$ were considered to be differentially targetable. Full scripts for piRNA target analysis available in the Supplemental Materials.

\section{Data availability}

Fly lines and antibodies are available from their original commercial or lab source. AntiPiwi 4K5 antibody is available from the Haifan Lin lab upon request. RNA-seq and Small RNA- 
seq sequence data are available at the National Center for Biotechnology Information GEO database with the accession number GSE171951.

\section{RESULTS}

\section{Maternal Piwi persists in germ cells throughout embryogenesis}

To begin investigating the function of maternal Piwi, we first characterized its expression pattern in embryos using the cross depicted in Fig 1A. Transheterozygous myc-piwi/UASp-GFP ( $3^{\text {rd }}$ chromosome) females were crossed with homozygous zfh2-gal4 ( $3^{\text {rd }}$ chromosome) males that express GAL4 throughout the nervous system (Jenett et al. 2012; Lai et al. 1991). The UASp-GFP/zfh2-gal4 progeny inherit maternal Myc-Piwi protein and express GFP but do not express Myc-Piwi from the zygotic genome. In contrast, myc-piwi/zfh2-gal4 progeny inherit maternal Myc-Piwi and express zygotic Myc-Piwi, but not GFP. With this approach, we were able to definitively identify UASp-GFP/zfh2-ga/4 embryos and use anti-Myc immunostaining to visualize maternal Piwi persistence during embryogenesis.

We observed strong maternal Myc-Piwi expression in UASp-GFP/zfh2-gal4 embryos up to at least 17 hours after egg laying (Fig 1B, left panels). At these relatively late stages of embryogenesis, maternal Myc-Piwi was detectable in a number of cell types, but most strongly in primordial germ cells (PGCs) (Fig 1B, left panels). As expected, Myc-Piwi was also strongly detectable in the PGCs of myc-piwi/zfh2-gal4 embryos, in which Myc-Piwi represents a mixture of maternal and zygotic Piwi (Fig 1B, middle panels). From PGC specification through migration, maternal Myc-Piwi localized within both the cytoplasm and nucleus, as previously described for maternal Piwi in early PGCs (Mani et al. 2014), but become predominantly nuclear in coalesced gonads (Fig 1C). These observations suggest that maternal Piwi could have a direct role in germline development throughout embryogenesis, including germline processes that occur during or after gonad coalescence. 
To visualize maternal Piwi in the larval gonad, we used a similar strategy, this time crossing transheterozygous myc-piwi/UASp-GFP females to Act-gal4 males (Fig S1). In UASpGFP/Act-gal4 third instar larvae in which Myc-Piwi represents only maternal Piwi, all testes and most ovaries lacked detectable maternal Myc-Piwi, but about $20 \%$ of ovaries did contain detectable maternal Myc-Piwi (Fig S1). This suggests that maternal Piwi is present during some stages of female larval gonadogenesis, up to the third instar stage.

\section{Knockdown of maternal piwi resulted in female-specific infertility in the F1 generation}

To assess the role of maternal Piwi in the germline development of progeny, we used Maternal Alpha Tubulin (MAT)-gal4 and two independent anti-piwi shRNA lines to reduce the levels of maternal Piwi (herein referred to as piwi-MatKD \#1 and \#2; Fig 2A). In these females (F0 females in Fig 2A), Piwi is knocked down specifically in germ cells starting in mid-oogenesis (Fig 2B). In contrast to strong piwi-null mutations where piwi expression in all ovarian cells is depleted, knocking down piwi expression in the germline did not affect germline stem cell selfrenewal and resulted in F0 ovaries with grossly normal morphology (Fig 2B). When piwi-MatKD F0 females are crossed with $w^{1118}$ males, half of the F1 progeny carries a copy of UASp-piwishRNA and the other half carries a copy of MAT-gal4 (Fig 2A). MatKD F1 flies used throughout this study were a mixture of these two genotypes. Although the maternal deposition of GAL4 protein could in theory activate the shRNA in the half of F1 progeny that carry UASp-shRNA (Staller et al. 2013), we saw barely detectable levels of zygotic Piwi protein and RNA during early- and mid-embryogenesis (Fig S2), so even if piwi-shRNA could reduce zygotically expressed piwi, the overall impact of this reduction as compared to the knockdown of maternal Piwi would be negligible.

Both anti-piwi shRNA lines efficiently reduced Piwi protein levels, but to different degrees: piwi-MatKD \#1 was relatively weaker than piwi-MatKD \#2 (Fig 2C). Accordingly, embryos laid by piwi-MatKD \#1 (“piwi MatKD \#1 F1” embryos) developed largely normally, 
whereas piwi-MatKD \#2 F1 embryos frequently arrested at pre-cellularization stages (Fig S3). This piwi-MatKD \#2 F1 phenotype is reminiscent of the F1 embryonic arrest that results from maternal Piwi depletion using strong piwi-null ovarian clones (Mani et al. 2014). Despite the high rate of embryonic arrest, some piwi-MatKD \#2 F1 embryos did progress to adulthood (see below).

We performed fertility tests of piwi-MatKD F0, F1, and F2 flies by crossing them individually with 2-3 $w^{1118}$ flies of the reciprocal sex, counting the number of eggs laid over seven days and the total number of adults that emerged from these vials after 12-15 days (Fig 2D-G). In contrast to piwi-null females, piwi-MatKD F0 females had normal ovary morphology (Fig 2B) and normal egg-laying capacity (Fig 2D, upper panel). In fact, they laid more eggs than control GFP-MatKD F0 females, possibly because the GFP-MatKD F0 females were somewhat compromised in egg-laying (Fig 2D, upper panel). Up to $100 \%$ of piwi-MatKD \#1 F1 embryos and up to $60 \%$ of piwi-MatKD \#2 F1 progressed to adulthood (Fig 2D, lower panel), consistent with the incomplete penetrance of embryonic arrest in piwi-MatKD F1 \#2 embryos (Fig S3). These F1 adults provided us with an opportunity to observe additional developmental defects in piwi-MatKD F1 flies.

Because we had observed that maternal Piwi was specifically maintained in the F1 embryonic germline (Fig 1), our measurement of the fertility of piwi-MatKD F1 females allowed us to assess whether maternal Piwi had any effect on progeny germline development. Indeed, piwi-MatKD F1 females from both anti-piwi shRNA lines suffered dramatic fertility defects (Fig $2 \mathrm{E}$, Fig S4). This was reflected in both decreased rates of egg-laying (Fig 2E, upper panel) and decreased percentages of those eggs developing to adulthood (Fig 2E, lower panel), as compared to GFP-MatKD F1 controls. Notably, although approximately $60 \%$ of piwi-MatKD \#2 F1 embryos could not progress beyond the first few cell cycles of embryogenesis (Fig S3), piwiMatKD \#2 F1 adult females were equally subfertile as piwi-MatKD \#1 F1 females (Fig 2E). 
If the piwi-MatKD F1 subfertility was caused by some general germline development defect, such as failure to specify PGCs during embryogenesis (Megosh et al. 2006), the fertility of both females and males would be affected. To our surprise, piwi-MatKD F1 males, when crossed with virgin $w^{1118}$ females, were able to stimulate egg laying (Fig $2 \mathrm{~F}$, upper panel) and fertilize those eggs (Fig 2F, lower panel) at rates indistinguishable from those of GFP-MatKD F1 males. The normal fertility of piwi-MatKD F1 males, together with the subfertility of piwi-MatKD F1 females, suggests that maternal Piwi plays some role in female-specific, rather than general, aspects of germline development in progeny.

We did not observe any subfertility in females in the piwi-MatKD F2 generation (Fig 2G, Fig S4, Fig S5), suggesting that zygotic piwi expression in the piwi-MatKD F1 ovary provides sufficient maternal Piwi for the normal germline development of piwi-MatKD F2 females. However, these F2 females were the progeny of the more mildly affected (not fully sterile) F1 females, and it is impossible to examine the phenotype of the progeny of severely affected (fully sterile) F1 females. Because of this, our measurement of F2 fertility is biased towards mildly affected females, and it remains unknown whether the defects of piwi-MatKD F1 females would be transmitted to their progeny.

To investigate what ovarian defects led to the subfertility of piwi-MatKD F1 females, we examined MatKD F1 gonads. Approximately $40 \%$ of piwi-MatKD F1 ovaries lacked late-stage egg chambers (“Arrested”) (Fig 3A, 3B). In these ovaries, oogenesis arrested at the onset of vitellogenesis (Fig 3B), a checkpoint where oogenesis can be stalled by a variety of stimuli ranging from environmental stress to imbalances in ecdysone or Juvenile Hormone levels (McCall 2004). Furthermore, 2-7\% of piwi-MatKD F1 ovaries completely lacked germline cells ("Agametic") (Fig 3A, 3B), a phenotype that evokes piwi-null mutant ovaries (Cox et al. 1998). The remaining $51-58 \%$ of piwi-MatKD F1 ovaries were morphologically wildtype-like (Fig 3A, 3B). These females with wildtype-like ovaries were nevertheless subfertile in comparison to GFP-MatKD F1 females (Fig S6), so the ovarian arrest was not the sole cause of piwi-MatKD 
F1 subfertility. All piwi-MatKD F1 ovaries had normal Piwi expression in both somatic and germline cells in non-degenerating egg chambers (Fig 3B), indicating that zygotic Piwi expression was not affected in piwi-MatKD F1 females.

The testes of piwi-MatKD F1 males had no observable defects (Fig 3C), which further reinforces the female-specific nature of the F1 fertility defect.

\section{Transposons are mildly derepressed in piwi-MatKD F1 embryos and fully repressed in piwi-MatKD F1 adult ovaries}

Given that the best-studied role of Piwi is in transposon repression, we next examined whether the piwi-MatKD F1 subfertility was a result of transposon derepression in the F1 embryo or the F1 adult ovary. To do so, we began by performing total RNA-seq on piwi-MatKD F1 0-1.5 h embryos (Fig S7, Table S1) and quantifying the expression of annotated transposons (Lerat et al. 2017). Only 11 transposons were derepressed under both piwi-MatKD conditions, with 16 derepressed in piwi-MatKD \#1 F1 and 34 derepressed in piwi-MatKD \#2 F1 embryos (Fig 4A, 4B). Among the derepressed transposons, retrotransposons in the LTR and LINE families were over-represented (Fig 4C). The expression of most of these derepressed transposons remained at relatively low levels, with the exception of Het-A (Fig S8), as compared to the typical derepression by 1-2 orders of magnitude for dozens of transposons when the Piwi/piRNA function is strongly disrupted (Rozhkov et al. 2013; Senti et al. 2015; Sytnikova et al. 2014).

To investigate whether this moderate transposon derepression in the early embryo could seed further transposon derepression later in development, we used RT-qPCR to assess transposon expression in MatKD F1 ovaries. We separated the wildtype-like and arrested ovaries so we could confidently compare gene expression in ovaries of similar cellular composition (GFP-MatKD F1 and piwi-MatKD F1 with wildtype-like ovaries) while also identifying potential expression differences between wildtype-like and arrested ovaries of the 
same maternal genotype. We observed no consistent derepression of the transposons that had been moderately derepressed in the early piwi-MatKD F1 embryo (Het-A, gypsy2, gypsy12, $T A R T$, and TAHRE) nor of those that were derepressed upon zygotic Piwi knockdown during embryogenesis (Het-A and HMS Beagle) as previously reported (Akkouche et al. 2017) (Fig 4D). In a few cases (Het-A, TART, gypsy12), there was mild derepression in some but not all the piwi-MatKD groups, but their expression was still much lower than in zuc-null ovaries, where the piRNA pathway is truly disrupted. Thus, despite the mild transposon derepression in the early piwi-MatKD F1 embryo, their repression was restored by adulthood. These analyses of transposon RNA levels in embryo and adult stages suggest that the piwi-MatKD F1 subfertility was not mainly caused by transposon derepression and led us to explore whether it could instead have been caused by the mis-regulation of non-transposon genes.

\section{The maternally deposited mRNA and piRNA pools are shifted by maternal piwi knockdown}

There is growing evidence that the PIWI/piRNA pathway also targets and regulates the expression of non-transposon genes (Wang and Lin 2021). As such, we used our RNA-seq of early F1 embryos to investigate whether piwi-MatKD had affected the broader maternally deposited transcriptome. Many more genes were differentially expressed in piwi-MatKD \#2 than in piwi-MatKD \#1 (Fig 5A). Many of the piwi-MatKD \#2 differentially expressed genes function in global proteolysis and apoptosis (Table S2), which likely reflects the increased propensity of piwi-MatKD \#2 F1 embryos to experience cell cycle defects and embryonic arrest (Fig S3) (Utz and Anderson 2000). Among the genes that were differentially expressed in both piwi-MatKD \#1 and \#2, 10 (including piwi) were down-regulated and 11 were up-regulated (Fig 5A, Table S3). As with all our analyses, we focused our attention on these gene expression changes in common between piwi-MatKD \#1 and piwi-MatKD \#2, because these are the most likely to explain the F1 subfertility that is in common between these two knockdown lines. Of the 21 
genes commonly differentially expressed in the piwi-MatKD early embryo (Table S3), none was clearly related to the female-specific F1 subfertility, with the possible exception of Jheh3 (see below).

Because we had observed maternal Piwi expression in the germline throughout embryogenesis (Fig 1), we next asked whether piwi-MatKD might cause the F1 infertility by dysregulating PIWI/piRNA-targeted genes in later developmental stages. To begin probing this possibility, we assessed the maternally deposited piRNA pool in MatKD F1 early embryos, reasoning that loss or accumulation of particular piRNAs in the early embryo could shape subsequent Piwi-mediated gene regulation throughout embryogenesis. We sequenced small RNAs isolated from total RNA of 0-1.5 h embryos (Fig 5B, Table S4) and found that the piRNA pool was reduced, though not abolished, by piwi-MatKD (Fig 5C). There was a particular reduction of 26-29 nucleotide piRNAs (Fig 5C), which are preferentially bound by Piwi rather than Aub or Ago3 (Brennecke et al. 2007). The remaining piRNAs in piwi-MatKD F1 embryos still largely retained the typical $1 \mathrm{U}$ and $10 \mathrm{~A}$ biases, though the former was slightly diminished while the latter was slightly enhanced compared to piRNAs from GFP-MatKD F1 embryos (Fig 5D). These changes in the size distribution, $1 \mathrm{U}$ bias, and $10 \mathrm{~A}$ bias suggest a shift from primary piRNAs to secondary piRNAs, which is consistent with a selective decrease of Piwi-bound piRNAs in the piwi-MatKD F1 early embryo.

To explore the possibility that these changes in the maternally deposited piRNA pool could impact PIWI/piRNA target genes later in development, we predicted target genes of the maternally deposited piRNAs. To guide PIWI proteins to a particular target, a piRNA must be able to base-pair with that RNA, though it does not need to base-pair perfectly (Barckmann et al. 2015; Gou et al. 2014; Halbach et al. 2020; Shen et al. 2018; Zhang et al. 2018). To identify target mRNAs, piRNA reads were aligned antisense to transcribed regions of non-transposon genes with up to two mismatches allowed. Each alignment was then weighted by the number of putative target sites of that piRNA read within the transcriptome. We refer to this calculated 
number of piRNA target sites within a gene as the "piRNA targetability" of that gene (see Methods and Supplemental Material for details of this calculation). Although this type of analysis has a limited ability to precisely identify specific target genes, it can give us a general sense of whether PIWI/piRNA have an altered capacity to target and regulate gene expression in piwiMatKD F1 embryos.

According to this analysis, approximately $50 \%$ of piRNA reads in all our libraries had the capacity to target at least one mRNA (Fig 5E). 63 genes had decreased piRNA targetability in piwi-MatKD compared to GFP-MatKD (Fig 5F, Table S5); that is, piRNAs antisense to these genes were abundant in GFP-MatKD control embryos, but were depleted in piwi-MatKD embryos. A few of these genes with changed piRNA targetability also had changed mRNA levels according to our RNA-seq. For example, the Juvenile Hormone Epoxide Hydrolase genes Jheh2 and Jheh3 had decreased piRNA targetability and increased mRNA expression (Fig 5G), indicating that their expression is normally repressed by Piwi and piRNAs. Juvenile Hormone impacts a myriad of processes in Drosophila, including longevity, stress response, and, notably, oogenesis (Santos et al. 2019). The Jheh gene family has not been studied extensively, but Jheh2 is known to be expressed in male, but not female, embryonic gonads (Casper and Van Doren 2009). The observation that maternal Piwi and piRNAs can repress a gene that is specifically expressed in male PGCs led us to hypothesize that maternal Piwi could regulate other sex-biased germline genes, a mechanism that could explain the subfertility of piwi-MatKD F1 females.

\section{The piwi-MatKD F1 female germline is partially masculinized}

Male-specific and female-specific characteristics of XY and XX PGCs, respectively, become evident around gonad coalescence (Casper and Van Doren 2009; Poirie et al. 1995; Staab et al. 1996; Wawersik et al. 2005), precisely the stage where we saw maternal Piwi protein was still maintained (Fig 1). In light of this and the fertility defects observed in piwi- 
MatKD F1 females, we asked whether female PGCs in piwi-MatKD F1 embryos were masculinized.

One key difference between male and female PGCs is the timing of their proliferation. PGCs form before cellularization of the embryo (Poirie et al. 1995). Normal numbers of PGCs were specified in piwi-MatKD F1 as compared to GFP-MatKD F1 embryos (Fig S9). After this initial phase, PGCs do not proliferate during migration to the embryonic gonad (Su et al. 1998), but male PGCs recommence proliferation at gonad coalescence, while female PGCs remain quiescent (Poirie et al. 1995; Wawersik et al. 2005). We saw this pattern reflected in GFPMatKD F1 embryos: there were similar numbers of PGCs in male and female embryos at stage 15, just after gonad coalescence, but at stages 16 and 17, male PGCs increased in number while female PGCs did not (Fig 6A). In contrast, in female piwi-MatKD F1 embryos, PGC numbers increased at stages 16 and 17, resembling their sibling male embryos (Fig 6B-C). This change indicates that PGCs in female piwi-MatKD F1 embryos acquired a male PGC proliferation pattern.

To explore whether this apparent masculinization of the piwi-MatKD F1 female germline continued in the adult ovary, we used RT-qPCR to examine the expression of adult ovarian mRNAs. We did not see any consistent change in the expression of canonical ovary-specific genes in piwi-MatKD F1 ovaries (Fig S10). However, some canonical testis-specific genes were present at higher levels in piwi-MatKD F1 ovaries than in controls (Fig 6D). These genes included always early (aly), a core factor in spermatid differentiation (White-Cooper et al. 2000), and fuzzy onion (fzo), which is activated by aly during spermatogenesis (Chen et al. 2011) (Fig $6 \mathrm{D}$, left panel). We also measured the expression of $p h f 7$, a histone reader important for spermatogenesis, and $p h f 7^{R C}$, the male-specific isoform of $p h f 7$ which causes oogenic arrest when overexpressed (Smolko et al. 2020). We observed that although the expression of phf7 is overall decreased in piwi-MatKD F1 ovaries, the relative proportion of $p h f 7^{R C}$ was increased in piwi-MatKD F1 arrested ovaries as compared to GFP-MatKD F1 ovaries (Fig 6D, right panels). 
The fact that only a subset of testis-specific genes we tested was derepressed in piwi-MatKD F1 ovaries is consistent with the fact that these ovaries do not have the classic tumor-like morphology of a dramatically masculinized female germline (Chau et al. 2009; Nagoshi et al. 1995; Steinmann-Zwicky et al. 1989) (Fig 3). Instead, we believe this represents a partial masculinization of the piwi-MatKD F1 ovary. We did not see defects in piwi-MatKD F1 somatic sex determination (Fig S11), so the partial masculinization in piwi-MatKD progeny appears to be germline-specific.

\section{DISCUSSION}

In this paper, we have reported that maternal Piwi has long-ranging functions on the development of progeny. Maternal Piwi can be detected in primordial germ cells (PGCs) throughout embryogenesis, and in at least some third instar larval ovaries. Furthermore, our analyses revealed a novel function of maternal Piwi in ensuring the germline development of female progeny. Our transcriptome analysis, piRNA analysis, and examination of embryonic germ cell number indicate a partial masculinization of the piwi-MatKD F1 female germline that is known to cause oogenic defects and sterility (Chau et al. 2009; Nagoshi et al. 1995; Schupbach 1985; Steinmann-Zwicky et al. 1989; Yang et al. 2012). These findings reveal a novel sexspecific function of a maternal-effect gene.

\section{A novel female-specific role for a maternal factor}

There have been decades of extensive study into maternal mutations that affect germline development in Drosophila, but most of these have focused on defects that manifest during early embryogenesis, such as the specification of PGCs (Schupbach and Wieschaus 1986; Seydoux and Braun 2006). Other studies have identified maternal-effect phenotypes that manifest in later PGC processes, such as migration through the primordial midgut (AsaokaTaguchi et al. 1999; DeGennaro et al. 2011; Kunwar et al. 2003) and survival during migration 
(Kugler et al. 2013; Slaidina and Lehmann 2017). In the case of maternal Piwi, we observed its persistence in PGCs through gonad coalescence (Fig 1), implying that it can impact even late embryonic stages of germline development.

Indeed, we saw that maternal piwi knockdown caused defects in the embryonic coalesced gonads (Fig 6) as well as adult gonads (Fig 2, 3) of progeny. Strikingly, the defects in fertility and gonadal morphology in piwi-MatKD F1s were specific to females. This could reflect that the female germline has a more stringent requirement for Piwi levels than the male germline, which has previously been suggested for zygotically-expressed Piwi (Gonzalez et al. 2015), or that maternal Piwi is specifically required for the oogenesis of female progeny. Nevertheless, to our knowledge, no maternal factor has previously been shown to specifically affect the development of one sex but not the other.

Germline development of males and females is largely similar during embryogenesis

until gonad coalescence, when sex-specific germline gene expression begins (Casper and Van Doren 2009) and male germ cells begin to proliferate while female germ cells remain quiescent (Poirie et al. 1995; Wawersik et al. 2005). Few studies, if any, have examined maternal-effect phenotypes at these late stages of embryogenesis, let alone in larval or adult stages. In light of our findings about maternal Piwi, it is likely that examining other maternal proteins for their longevity and functions beyond embryogenesis will reveal new developmental roles long after the maternal-to-zygotic transition.

\section{The embryonic PIWI/piRNA pathway and transposon control}

Since early observations that maternal PIWI proteins and piRNAs accumulate in the germ plasm (Brennecke et al. 2007; Harris and Macdonald 2001; Megosh et al. 2006), it has been accepted that the maternal deposition of piRNAs shapes the piRNA pool going forward in development. So far, examination of this hypothesis has been focused on the molecular mechanisms of piRNA biogenesis and piRNA involvement in epigenetic regulation and 
transposon suppression (Akkouche et al. 2013; Brennecke et al. 2008; de Vanssay et al. 2012; Gu and Elgin 2013; Le Thomas et al. 2014a; Le Thomas et al. 2014b). In addition to directly binding and regulating zygotic genes, maternally deposited piRNAs can contribute to the generation of zygotic piRNAs via the secondary piRNA biogenesis pathway once zygotic mRNA substrates begin to be produced (Barckmann et al. 2018; Wang et al. 2015). For example, in Bombyx mori, maternally deposited piRNAs are stable for at least 12 hours during embryogenesis and trigger the generation of new piRNAs when they target zygotic mRNAs (Kawaoka et al. 2011). This means that loss of maternal piRNAs can affect gene expression beyond the maternal phase of embryogenesis. The best-characterized examples of this to date are when piRNAs targeting paternally-inherited transposons are absent in the maternal pool, resulting in transposon derepression and atrophic or arrested ovaries in adults (Akkouche et al. 2013; Brennecke et al. 2008). Our piwi-MatKD strategy resulted in a reduced pool of Piwi-bound piRNAs in the early embryo (Fig 5C), which could reflect either a disruption of piRNA biogenesis during oogenesis or instability of piRNAs unbound by their partner PIWI protein, or both. Regardless, this loss of maternal piRNAs almost certainly would change PIWI/piRNA target RNAs later in embryogenesis.

Despite the reduced pool of maternally deposited piRNAs, piwi-MatKD F1 individuals did not experience the dramatic derepression of transposons that is emblematic of disruptions to the PIWI/piRNA pathway (Fig 4). Although several transposons were consistently derepressed in piwi-MatKD F1 early embryos (Fig 4A), most of these still remained at fairly low expression levels (Fig S8). The exception was a group of telomere-associated transposons: Het- $A$, TART, and TAHRE. Derepression of these transposons during oogenesis can cause early embryonic chromosome segregation defects (Durdevic et al. 2018; Morgunova et al. 2015) that resemble defects of embryos strongly depleted of maternal Piwi (Mani et al. 2014), so the up-regulation of these transposons in piwi-MatKD \#2 F1 embryos may partially explain their frequent embryonic arrest (Fig S3). However, it is unclear how the observed mild derepression of Het-A, TART, and 
TAHRE in the early embryo might relate to the female-specific piwi-MatKD F1 subfertility which manifested in adulthood, especially because these transposons were repressed in piwi-MatKD F1 adult ovaries (Fig 4D). If Het-A, TAHRE, and TART contributed to the piwi-MatKD F1 female subfertility, it must have been indirectly through transient activity.

Previous studies have shown that the PIWI/piRNA pathway is actively involved in transposon repression in gonads throughout development (Marie et al. 2017), and the transient knockdown of zygotic Piwi during mid- to late- embryogenesis (3-16 hours after egg laying) results in massive transposon derepression and subsequent sterility in adult ovaries (Akkouche et al. 2017). While the knockdown strategy used by Akkouche et al. reduced piwi mRNA levels during embryogenesis, it did not affect levels of maternal Piwi protein, which we have shown to remain present at high levels throughout embryogenesis (Fig 1). In contrast, because we saw that zygotic Piwi was barely detectable at mid-embryogenesis (Fig S2), any effect on zygotic piwi expression was likely negligible compared to the effect on maternal piwi expression in our knockdown strategy. Furthermore, our knockdown strategy reduced maternal Piwi protein levels starting from mid-oogenesis in F0 females (Fig 2A-B). As such, piwi-MatKD F1 embryos not only lacked the normal function of maternal Piwi during embryogenesis, but also inherited any dysregulation of gene expression (Fig 5A), piRNA biogenesis (Fig 5C-F), and possibly epigenetic state that occurred during oogenesis. Both of these embryonic Piwi knockdown strategies ultimately resulted in subfertile adult females, but the different effects on transposon repression in these two systems suggest that this subfertility is produced by different mechanisms. Akkouche et al. did not discuss the fertility of males depleted of zygotic Piwi. Given our report of female-specific germline defects upon maternal piwi knockdown, examining the males depleted of zygotic Piwi during embryogenesis will aid in the further disentanglement of maternal and zygotic Piwi functions during embryogenesis.

\section{The PIWI/piRNA pathway and sex determination}


There is a growing understanding that the regulatory potential of the PIWI/piRNA pathway extends far beyond transposon suppression (Rojas-Rios et al. 2017; Wang and Lin 2021). This is especially well-understood in C. elegans and mice and is becoming increasingly apparent in Drosophila in the case of Aubergine (Barckmann et al. 2015; Ramat et al. 2020; Rouget et al. 2010; Vourekas et al. 2016). Given the extreme heterogeneity and low complementarity requirements of piRNAs (Barckmann et al. 2015; Gou et al. 2014; Halbach et al. 2020; Shen et al. 2018; Zhang et al. 2018), it is not surprising that they can target and regulate a wide variety of RNAs.

In the context of this study, perhaps the most relevant examples of PIWI proteins regulating non-transposon mRNAs are in the cases of sex determination in $B$. mori and $C$. elegans. Despite otherwise very different modes of sex determination, individual piRNAs have been implicated in this process for both organisms. In B. mori, the Fem piRNA targets the mRNA of male sex determination gene Masc for degradation in females (Kiuchi et al. 2014), while in C. elegans, the $21 u x-1$ piRNA targets the mRNA of male sex determination gene xol-1 for degradation in hermaphrodies (Tang et al. 2018). When these piRNAs were deleted, the organisms became partially masculinized. Although we did not identify specific piRNAs involved in sex determination in Drosophila in this study, the partial masculinization of piwi-MatKD F1 female PGCs and ovaries (Fig 6) suggests a role for the Drosophila PIWI/piRNA pathway in repressing male gene expression programs in the female germline.

Both in C. elegans and in our study, modulation of PIWI proteins and/or piRNAs did not fully transform XX individuals into males, but rather moderately masculinized them (Tang et al. 2018) (Fig 6), implying a partial shift from female gene expression patterns to male gene expression patterns. This emerging link between the PIWI/piRNA pathway and sex determination may be yet another illustration of how piRNAs can be cellular tools to "tune," rather than completely switch, gene expression programs. 


\section{ACKNOWLEDGEMENTS}

We thank the Developmental Studies Hybridoma Bank for providing antibodies, and the Bloomington Drosophila Stock Center, Drs. Ting Xie, Trudi Schupbach, and Mark van Doren for providing fly stocks. We thank Drs. Mark van Doren, Nils Neuenkirchen, Hongying Qi, Valerie Reinke, Lynn Cooley, and Karla Neugebauer for discussion and technical support, and Drs. Celina Juliano, Nils Neuenkirchen, Hongying Qi, and Valerie Reinke for valuable comments on the manuscript. Dr. Nils Neuenkirchen also wrote our custom script (available in Supplemental Materials) for adding an $\mathrm{NH}$ tag to Bowtie output files in the piRNA sequencing analysis.

Sequencing service was conducted at the Yale Stem Cell Center Genomics Core facility, which was supported by the Connecticut Regenerative Medicine Research Fund and the Li Ka Shing Foundation.

Author Contributions: LG and HL conceived and designed the project. LG conducted all experiments and analysis, except XT conducted and analyzed larval gonad immunofluorescence experiments in Fig S1. LG prepared the figures and the initial draft. LG, $H L$, and XT revised the paper. HL supervised the study.

Funding: This research was supported by a gift from Luye Life Sciences to HL. LG was supported by NSF Graduate Research Fellowship Program (DGE1752134) and the Training Program in Genetics (NIH T32 GM007499).

\section{REFERENCES}

Akkouche A, Grentzinger T, Fablet M, Armenise C, Burlet N, Braman V, Chambeyron S, Vieira C. 2013. Maternally deposited germline pirnas silence the tirant retrotransposon in somatic cells. EMBO Rep. 14(5):458-464. 
Akkouche A, Mugat B, Barckmann B, Varela-Chavez C, Li B, Raffel R, Pelisson A, Chambeyron S. 2017. Piwi is required during drosophila embryogenesis to license dual-strand pirna clusters for transposon repression in adult ovaries. Mol Cell. 66(3):411-419 e414.

Anders S, Huber W. 2010. Differential expression analysis for sequence count data. Genome Biol. 11(10):R106.

Andrews S. 2010. Fastqc: A quality control tool for high throughput sequence data. Available online at: $\underline{\mathrm{Http}: / / w w w . B i o i n f o r m a t i c s . B a b r a h a m . A c . U k / p r o j e c t s / f a s t q c}$

Aravin A, Gaidatzis D, Pfeffer S, Lagos-Quintana M, Landgraf P, lovino N, Morris P, Brownstein MJ, Kuramochi-Miyagawa S, Nakano T et al. 2006. A novel class of small rnas bind to mili protein in mouse testes. Nature. 442(7099):203-207.

Asaoka-Taguchi M, Yamada M, Nakamura A, Hanyu K, Kobayashi S. 1999. Maternal pumilio acts together with nanos in germline development in drosophila embryos. Nat Cell Biol. $1(7): 431-437$.

Barckmann B, Dufourt J, Simonelig M. 2018. Iclip of the piwi protein aubergine in drosophila embryos. Methods Mol Biol. 1720:89-110.

Barckmann B, Pierson S, Dufourt J, Papin C, Armenise C, Port F, Grentzinger T, Chambeyron S, Baronian G, Desvignes JP et al. 2015. Aubergine iclip reveals pirna-dependent decay of mrnas involved in germ cell development in the early embryo. Cell Rep. 12(7):12051216.

Brennecke J, Aravin AA, Stark A, Dus M, Kellis M, Sachidanandam R, Hannon GJ. 2007. Discrete small rna-generating loci as master regulators of transposon activity in drosophila. Cell. 128(6):1089-1103.

Brennecke J, Malone CD, Aravin AA, Sachidanandam R, Stark A, Hannon GJ. 2008. An epigenetic role for maternally inherited pirnas in transposon silencing. Science. 322(5906):1387-1392. 
Casper AL, Van Doren M. 2009. The establishment of sexual identity in the drosophila germline. Development. 136(22):3821-3830.

Chau J, Kulnane LS, Salz HK. 2009. Sex-lethal facilitates the transition from germline stem cell to committed daughter cell in the drosophila ovary. Genetics. 182(1):121-132.

Chen X, Lu C, Morillo Prado JR, Eun SH, Fuller MT. 2011. Sequential changes at differentiation gene promoters as they become active in a stem cell lineage. Development. 138(12):2441-2450.

Cox DN, Chao A, Baker J, Chang L, Qiao D, Lin H. 1998. A novel class of evolutionarily conserved genes defined by piwi are essential for stem cell self-renewal. Genes Dev. 12(23):3715-3727.

Cox DN, Chao A, Lin H. 2000. Piwi encodes a nucleoplasmic factor whose activity modulates the number and division rate of germline stem cells. Development. 127(3):503-514.

de Vanssay A, Bouge AL, Boivin A, Hermant C, Teysset L, Delmarre V, Antoniewski C, Ronsseray S. 2012. Paramutation in drosophila linked to emergence of a pirnaproducing locus. Nature. 490(7418):112-115.

DeGennaro M, Hurd TR, Siekhaus DE, Biteau B, Jasper H, Lehmann R. 2011. Peroxiredoxin stabilization of de-cadherin promotes primordial germ cell adhesion. Dev Cell. 20(2):233243.

Dobin A, Davis CA, Schlesinger F, Drenkow J, Zaleski C, Jha S, Batut P, Chaisson M, Gingeras TR. 2013. Star: Ultrafast universal rna-seq aligner. Bioinformatics. 29(1):15-21.

Dorogova NV, Bolobolova EU, Zakharenko LP. 2017. Cellular aspects of gonadal atrophy in drosophila p-m hybrid dysgenesis. Dev Biol. 424(2):105-112.

Dufourt J, Bontonou G, Chartier A, Jahan C, Meunier AC, Pierson S, Harrison PF, Papin C, Beilharz TH, Simonelig M. 2017. Pirnas and aubergine cooperate with wispy poly(a) polymerase to stabilize mrnas in the germ plasm. Nat Commun. 8(1):1305. 
Durdevic Z, Pillai RS, Ephrussi A. 2018. Transposon silencing in the drosophila female germline is essential for genome stability in progeny embryos. Life Sci Alliance. 1(5):e201800179. Engels WR, Preston CR. 1979. Hybrid dysgenesis in drosophila melanogaster: The biology of female and male sterility. Genetics. 92(1):161-174.

Girard A, Sachidanandam R, Hannon GJ, Carmell MA. 2006. A germline-specific class of small rnas binds mammalian piwi proteins. Nature. 442(7099):199-202.

Gonzalez J, Qi H, Liu N, Lin H. 2015. Piwi is a key regulator of both somatic and germline stem cells in the drosophila testis. Cell Rep. 12(1):150-161.

Gou LT, Dai P, Yang JH, Xue Y, Hu YP, Zhou Y, Kang JY, Wang X, Li H, Hua MM et al. 2014. Pachytene pirnas instruct massive mrna elimination during late spermiogenesis. Cell Res. 24(6):680-700.

Grivna ST, Pyhtila B, Lin H. 2006. Miwi associates with translational machinery and piwiinteracting rnas (pirnas) in regulating spermatogenesis. Proc Natl Acad Sci U S A. 103(36):13415-13420.

Gu T, Elgin SC. 2013. Maternal depletion of piwi, a component of the rnai system, impacts heterochromatin formation in drosophila. PLoS Genet. 9(9):e1003780.

Halbach R, Miesen P, Joosten J, Taskopru E, Rondeel I, Pennings B, Vogels CBF, Merkling SH, Koenraadt CJ, Lambrechts L et al. 2020. A satellite repeat-derived pirna controls embryonic development of aedes. Nature. 580(7802):274-277.

Harris AN, Macdonald PM. 2001. Aubergine encodes a drosophila polar granule component required for pole cell formation and related to eif2c. Development. 128(14):2823-2832.

Jemc JC. 2011. Somatic gonadal cells: The supporting cast for the germline. Genesis. 49(10):753-775.

Jenett A, Rubin GM, Ngo TT, Shepherd D, Murphy C, Dionne H, Pfeiffer BD, Cavallaro A, Hall D, Jeter J et al. 2012. A gal4-driver line resource for drosophila neurobiology. Cell Rep. 2(4):991-1001. 
Kawaoka S, Arai Y, Kadota K, Suzuki Y, Hara K, Sugano S, Shimizu K, Tomari Y, Shimada T, Katsuma S. 2011. Zygotic amplification of secondary pirnas during silkworm embryogenesis. RNA. 17(7):1401-1407.

Kidwell MG, Kidwell JF, Sved JA. 1977. Hybrid dysgenesis in drosophila melanogaster: A syndrome of aberrant traits including mutation, sterility and male recombination. Genetics. 86(4):813-833.

Kiuchi T, Koga H, Kawamoto M, Shoji K, Sakai H, Arai Y, Ishihara G, Kawaoka S, Sugano S, Shimada T et al. 2014. A single female-specific pirna is the primary determiner of sex in the silkworm. Nature. 509(7502):633-636.

Klein JD, Qu C, Yang X, Fan Y, Tang C, Peng JC. 2016. C-fos repression by piwi regulates drosophila ovarian germline formation and tissue morphogenesis. PLoS Genet. 12(9):e1006281.

Klenov MS, Sokolova OA, Yakushev EY, Stolyarenko AD, Mikhaleva EA, Lavrov SA, Gvozdev VA. 2011. Separation of stem cell maintenance and transposon silencing functions of piwi protein. Proc Natl Acad Sci U S A. 108(46):18760-18765.

Kotov AA, Adashev VE, Godneeva BK, Ninova M, Shatskikh AS, Bazylev SS, Aravin AA, Olenina LV. 2019. Pirna silencing contributes to interspecies hybrid sterility and reproductive isolation in drosophila melanogaster. Nucleic Acids Res. 47(8):4255-4271.

Kugler JM, Chen YW, Weng R, Cohen SM. 2013. Maternal loss of mirnas leads to increased variance in primordial germ cell numbers in drosophila melanogaster. G3 (Bethesda). 3(9):1573-1576.

Kunwar PS, Starz-Gaiano M, Bainton RJ, Heberlein U, Lehmann R. 2003. Tre1, a g proteincoupled receptor, directs transepithelial migration of drosophila germ cells. PLoS Biol. 1(3):E80. 
Lai ZC, Fortini ME, Rubin GM. 1991. The embryonic expression patterns of zfh-1 and zfh-2, two drosophila genes encoding novel zinc-finger homeodomain proteins. Mech Dev. 34(23):123-134.

Langmead B, Trapnell C, Pop M, Salzberg SL. 2009. Ultrafast and memory-efficient alignment of short DNA sequences to the human genome. Genome Biol. 10(3):R25.

Lau NC, Seto AG, Kim J, Kuramochi-Miyagawa S, Nakano T, Bartel DP, Kingston RE. 2006. Characterization of the pirna complex from rat testes. Science. 313(5785):363-367.

Le Thomas A, Marinov GK, Aravin AA. 2014a. A transgenerational process defines pirna biogenesis in drosophila virilis. Cell Rep. 8(6):1617-1623.

Le Thomas A, Stuwe E, Li S, Du J, Marinov G, Rozhkov N, Chen YC, Luo Y, Sachidanandam R, Toth KF et al. 2014b. Transgenerationally inherited pirnas trigger pirna biogenesis by changing the chromatin of pirna clusters and inducing precursor processing. Genes Dev. 28(15):1667-1680.

Lee HC, Gu W, Shirayama M, Youngman E, Conte D, Jr., Mello CC. 2012. C. Elegans pirnas mediate the genome-wide surveillance of germline transcripts. Cell. 150(1):78-87.

Lehmann R. 2016. Germ plasm biogenesis--an oskar-centric perspective. Curr Top Dev Biol. 116:679-707.

Lerat E, Fablet M, Modolo L, Lopez-Maestre H, Vieira C. 2017. Tetools facilitates big data expression analysis of transposable elements and reveals an antagonism between their activity and that of pirna genes. Nucleic Acids Res. 45(4):e17.

Liao Y, Smyth GK, Shi W. 2014. Featurecounts: An efficient general purpose program for assigning sequence reads to genomic features. Bioinformatics. 30(7):923-930.

Lin KY, Wang WD, Lin CH, Rastegari E, Su YH, Chang YT, Liao YF, Chang YC, Pi H, Yu BY et al. 2020. Piwi reduction in the aged niche eliminates germline stem cells via toll-gsk3 signaling. Nat Commun. 11(1):3147. 
Ma X, Zhu X, Han Y, Story B, Do T, Song X, Wang S, Zhang Y, Blanchette M, Gogol M et al. 2017. Aubergine controls germline stem cell self-renewal and progeny differentiation via distinct mechanisms. Dev Cell. 41(2):157-169 e155.

Mani SR, Megosh H, Lin H. 2014. Piwi proteins are essential for early drosophila embryogenesis. Dev Biol. 385(2):340-349.

Marie PP, Ronsseray S, Boivin A. 2017. From embryo to adult: Pirna-mediated silencing throughout germline development in drosophila. G3 (Bethesda). 7(2):505-516.

McCall K. 2004. Eggs over easy: Cell death in the drosophila ovary. Dev Biol. 274(1):3-14.

Megosh HB, Cox DN, Campbell C, Lin H. 2006. The role of piwi and the mirna machinery in drosophila germline determination. Curr Biol. 16(19):1884-1894.

Morgunova V, Akulenko N, Radion E, Olovnikov I, Abramov Y, Olenina LV, Shpiz S, Kopytova DV, Georgieva SG, Kalmykova A. 2015. Telomeric repeat silencing in germ cells is essential for early development in drosophila. Nucleic Acids Res. 43(18):8762-8773.

Nagoshi RN, Patton JS, Bae E, Geyer PK. 1995. The somatic sex determines the requirement for ovarian tumor gene activity in the proliferation of the drosophila germline. Development. 121(2):579-587.

Nishida KM, Saito K, Mori T, Kawamura Y, Nagami-Okada T, Inagaki S, Siomi H, Siomi MC. 2007. Gene silencing mechanisms mediated by aubergine pirna complexes in drosophila male gonad. RNA. 13(11):1911-1922.

Poirie M, Niederer E, Steinmann-Zwicky M. 1995. A sex-specific number of germ cells in embryonic gonads of drosophila. Development. 121(6):1867-1873.

Ramat A, Garcia-Silva MR, Jahan C, Nait-Saidi R, Dufourt J, Garret C, Chartier A, Cremaschi J, Patel V, Decourcelle M et al. 2020. The piwi protein aubergine recruits eif3 to activate translation in the germ plasm. Cell Res. 30(5):421-435. 
Rojas-Rios P, Chartier A, Pierson S, Simonelig M. 2017. Aubergine and pirnas promote germline stem cell self-renewal by repressing the proto-oncogene cbl. EMBO J. 36(21):3194-3211.

Rouget C, Papin C, Boureux A, Meunier AC, Franco B, Robine N, Lai EC, Pelisson A, Simonelig M. 2010. Maternal mrna deadenylation and decay by the pirna pathway in the early drosophila embryo. Nature. 467(7319):1128-1132.

Rozhkov NV, Hammell M, Hannon GJ. 2013. Multiple roles for piwi in silencing drosophila transposons. Genes Dev. 27(4):400-412.

Saito K, Nishida KM, Mori T, Kawamura Y, Miyoshi K, Nagami T, Siomi H, Siomi MC. 2006. Specific association of piwi with rasirnas derived from retrotransposon and heterochromatic regions in the drosophila genome. Genes Dev. 20(16):2214-2222.

Santos CG, Humann FC, Hartfelder K. 2019. Juvenile hormone signaling in insect oogenesis.

Curr Opin Insect Sci. 31:43-48.

Schupbach T. 1985. Normal female germ cell differentiation requires the female $\mathrm{x}$ chromosome to autosome ratio and expression of sex-lethal in drosophila melanogaster. Genetics. 109(3):529-548.

Schupbach T, Wieschaus E. 1986. Maternal-effect mutations altering the anterior-posterior pattern of the drosophila embryo. Rouxs Arch Dev Biol. 195(5):302-317.

Senti KA, Jurczak D, Sachidanandam R, Brennecke J. 2015. Pirna-guided slicing of transposon transcripts enforces their transcriptional silencing via specifying the nuclear pirna repertoire. Genes Dev. 29(16):1747-1762.

Seydoux G, Braun RE. 2006. Pathway to totipotency: Lessons from germ cells. Cell. 127(5):891-904.

Shen EZ, Chen H, Ozturk AR, Tu S, Shirayama M, Tang W, Ding YH, Dai SY, Weng Z, Mello CC. 2018. Identification of pirna binding sites reveals the argonaute regulatory landscape of the c. Elegans germline. Cell. 172(5):937-951 e918. 
Siddiqui NU, Li X, Luo H, Karaiskakis A, Hou H, Kislinger T, Westwood JT, Morris Q, Lipshitz HD. 2012. Genome-wide analysis of the maternal-to-zygotic transition in drosophila primordial germ cells. Genome Biol. 13(2):R11.

Slaidina M, Lehmann R. 2017. Quantitative differences in a single maternal factor determine survival probabilities among drosophila germ cells. Curr Biol. 27(2):291-297.

Smolko AE, Shapiro-Kulnane L, Salz HK. 2020. An autoregulatory switch in sex-specific phf7 transcription causes loss of sexual identity and tumors in the drosophila female germline. Development. 147(17).

Staab S, Heller A, Steinmann-Zwicky M. 1996. Somatic sex-determining signals act on xx germ cells in drosophila embryos. Development. 122(12):4065-4071.

Staller MV, Yan D, Randklev S, Bragdon MD, Wunderlich ZB, Tao R, Perkins LA, Depace AH, Perrimon N. 2013. Depleting gene activities in early drosophila embryos with the "maternal-gal4-shrna" system. Genetics. 193(1):51-61.

Steinmann-Zwicky M, Schmid H, Nothiger R. 1989. Cell-autonomous and inductive signals can determine the sex of the germ line of drosophila by regulating the gene sxl. Cell. 57(1):157-166.

Su TT, Campbell SD, O'Farrell PH. 1998. The cell cycle program in germ cells of the drosophila embryo. Dev Biol. 196(2):160-170.

Sytnikova YA, Rahman R, Chirn GW, Clark JP, Lau NC. 2014. Transposable element dynamics and piwi regulation impacts Incrna and gene expression diversity in drosophila ovarian cell cultures. Genome Res. 24(12):1977-1990.

Tang W, Seth M, Tu S, Shen EZ, Li Q, Shirayama M, Weng Z, Mello CC. 2018. A sex chromosome pirna promotes robust dosage compensation and sex determination in c. Elegans. Dev Cell. 44(6):762-770 e763.

Utz PJ, Anderson P. 2000. Life and death decisions: Regulation of apoptosis by proteolysis of signaling molecules. Cell Death Differ. 7(7):589-602. 
Vagin VV, Sigova A, Li C, Seitz H, Gvozdev V, Zamore PD. 2006. A distinct small rna pathway silences selfish genetic elements in the germline. Science. 313(5785):320-324.

Van Doren M, Williamson AL, Lehmann R. 1998. Regulation of zygotic gene expression in drosophila primordial germ cells. Curr Biol. 8(4):243-246.

Vourekas A, Alexiou P, Vrettos N, Maragkakis M, Mourelatos Z. 2016. Sequence-dependent but not sequence-specific pirna adhesion traps mrnas to the germ plasm. Nature. 531(7594):390-394.

Wang C, Lin H. 2021. Roles of pirnas in transposon and pseudogene regulation of germline mrnas and Incrnas. Genome Biol. 22(1):27.

Wang W, Han BW, Tipping C, Ge DT, Zhang Z, Weng Z, Zamore PD. 2015. Slicing and binding by ago3 or aub trigger piwi-bound pirna production by distinct mechanisms. Mol Cell. $59(5): 819-830$.

Wawersik M, Milutinovich A, Casper AL, Matunis E, Williams B, Van Doren M. 2005. Somatic control of germline sexual development is mediated by the jak/stat pathway. Nature. 436(7050):563-567.

White-Cooper H, Leroy D, MacQueen A, Fuller MT. 2000. Transcription of meiotic cell cycle and terminal differentiation genes depends on a conserved chromatin associated protein, whose nuclear localisation is regulated. Development. 127(24):5463-5473.

Yang SY, Baxter EM, Van Doren M. 2012. Phf7 controls male sex determination in the drosophila germline. Dev Cell. 22(5):1041-1051.

Zhang D, Tu S, Stubna M, Wu WS, Huang WC, Weng Z, Lee HC. 2018. The pirna targeting rules and the resistance to pirna silencing in endogenous genes. Science. 359(6375):587-592. 


\section{FIGURE LEGENDS}

Figure 1. Maternal Piwi persists in germ cells through gonad coalescence. (A) Schematic of crossing strategy to visualize maternal Piwi. (B) Representative images from visualization of Myc-Piwi in late-stage embryos (approximately 16 hours after egg laying) inheriting only maternal Myc-Piwi protein (left panel), inheriting both maternal Myc-Piwi protein and the mycpiwi transgene (middle panel), and inheriting neither the Myc-Piwi protein nor the myc-piwi transgene (right panel; negative control). Coalesced gonads indicated by arrowheads. (C) Representative images of maternal Myc-Piwi protein in UASp-GFP/zfh2-gal4 embryos from the cross depicted in Fig 1A, and schematics of germ cell localization at each developmental timepoint. Inset is magnified 2X. PGC: primordial germ cells.

Figure 2. Maternal Piwi is required for fertility of female, but not male, progeny. (A) Schematic of crossing strategy to deplete maternal Piwi. F0 individuals carry both UASp-shRNA and Maternal Alpha Tubulin (MAT)-GAL4, so shRNA is expressed from mid-oogenesis. F1 individuals carry either UASp-shRNA or MAT-GAL4, but not both. (B) Schematic of MAT-GAL4 expression in the MatKD F0 ovary, and anti-Piwi immunofluorescence in MatKD F0 ovaries. Whole ovary images are at the same scale. (C) Western blot for total Piwi and total GAPDH in 0-2 h F1 embryos. (D-G) Seven-day fertility tests of individual females mated to two $w^{1118}$ males or individual males mated to three $w^{1118}$ females (see cross in Fig 2A). (D) MatKD F0 females $(\mathrm{n}=17-25)$. (E) MatKD F1 females (n=16-22). (F) MatKD F1 males $(\mathrm{n}=17-20)$. (G) MatKD F2 females $(n=20-24)$. Upper panels indicate number of eggs laid per day and lower panels indicate the percentage of those eggs that developed to adulthood (only calculated for crosses which produced $\geq 10$ eggs). Mean \pm SD. One-way ANOVA and Dunnet's multiple comparisons test. n.s. = "not significant."

Figure 3. Adult females depleted of maternal Piwi had arrested ovaries. (A) Representative images of MatKD F1 ovaries (2-3 days old), and relative frequency of each category of ovary 
phenotype in each genotype ( $n=45-75$ per genotype). Chi-square test. Whole ovary images are at the same scale. (B) Anti-Vasa and anti-Piwi immunofluorescence of MatKD arrested ovaries. Piwi-MatKD arrested ovaries arrest around stage 8. An asterisk indicates one example mature egg, and an arrowhead indicates one example arrested egg chamber. (C) Representative images of MatKD F1 testes, with anti-Vasa and anti-Piwi immunofluorescence, and Differentially Interference Contrast (DIC) to visualize sperm tails.

Figure 4. Transposons were marginally derepressed in the piwi-MatKD F1 generation. (AB) Differential expression of transposons from RNA-seq on 0-1.5 h MatKD F1 embryos. Red datapoints are significantly changed ( $p$-adjusted $<0.05$, Fold Change $>1.5$ ) in that genotype compared to GFP-MatKD, and large red datapoints are significantly changed ( $p$-adjusted < 0.05, Fold Change > 1.5) in both piwi-MatKD \#1 and \#2 compared to GFP-MatKD. (C) Relative proportion of transposon classes in each group. (D) RT-qPCR for transposons in MatKD F1 adult ovaries, zuc $^{+-}\left(\right.$zucchini $\left.^{+/ H M 27}\right)$, and zuc $c^{-/-}\left(\right.$zucchini $\left.^{-H M 27 / D f(2 L) 716}\right)$ ovaries. All piwi-MatKD groups were compared to GFP-MatKD, and $z u c^{-/-}$was compared to $z u c^{+/}$. The latter comparison serves as a positive control for transposon derepression in the context of piRNA pathway disruption. Two-way ANOVA and Dunnett's Multiple Comparisons Test, ${ }^{*} p<0.05,{ }^{* *} p<0.01,{ }^{* * *}$ $p<0.0001$.

Figure 5. Knockdown of maternal piwi shifts the maternally deposited transcriptome and piRNA pool in the early F1 embryo. (A) Differential expression of non-transposon genes from RNA-seq on 0-1.5 h MatKD F1 embryos. Red datapoints are significantly changed (p-adjusted < 0.05, Fold Change > 1.5) in that genotype compared to GFP-MatKD, and large red datapoints are significantly changed ( $p$-adjusted $<0.05$, Fold Change $>1.5$ ) in both piwi-MatKD \#1 and \#2 compared to GFP-MatKD. (B) Small RNAs (20 - $29 \mathrm{nt})$ were isolated from total 0-1.5 h MatKD F1 embryo RNA for Small RNA-seq. (C) Size distribution of piRNAs from total Small RNA-seq, after filtering out rRNA, miRNA, and siRNA, normalized to total library size. Two-Way ANOVA, 
Dunnett's Multiple Comparisons Test. * $p<0.01,{ }^{* *} p<0.0001$. (D) Sequence distribution from each piRNA library at the first and tenth nucleotide position. (E) Percentage of reads in each piRNA library which have the capacity to target mRNAs, defined as being antisense to a transcribed gene region, allowing up to two mismatches. (F) Differential Targetability of Drosophila non-transposon mRNAs in piwi-MatKD vs GFP-MatKD F1 embryos. Red datapoints are significantly changed ( $p$-adjusted $<0.05$, Fold Change $>1.5$ ) in that genotype compared to GFP-MatKD, and large red datapoints are significantly changed ( $p$-adjusted $<0.05$, Fold Change > 1.5) in both piwi-MatKD\#1 and \#2 compared to GFP-MatKD. (G) Putative antisense piRNA levels (upper panel, from Small RNA-seq) and mRNA levels (lower panel, from RNAseq) for Jheh2 and Jheh3. Relative levels determined from DESeq2. Significance tested with Two-Way ANOVA and Dunnett's Multiple Comparisons Test.

Figure 6. The piwi-MatKD F1 female germline is partially masculinized. (A - C) Germ cell numbers in male and female embryonic MatKD F1 gonads (stages 15-17) were visualized (upper panel) and counted (lower panels). Embryos were sexed by paternally inherited Dfd-lacZ on the $\mathrm{X}$ chromosome and embryos were staged based on gut morphology. Violin plots indicate germ cell count at each stage and condition; thick dashed line indicates median and thin dotted line indicates $1^{\text {st }}$ and $3^{\text {rd }}$ quartiles. Line indicates regression line of germ cell numbers of each sex within each genotype, $\beta 1$ indicates the coefficient calculated from Poisson linear regression, and $\mathrm{R}^{2}$ indicates fit of the data to regression line. Asterisk indicates significance of the coefficient $\left({ }^{* *} p<0.01,{ }^{* * *} p<0.001\right)$ compared to the null hypothesis $(\beta 1=0) . n=66-81$ per sex of each genotype, with 20-27 per stage of each sex of each genotype. (A) GFP-MatKD F1 embryos. (B) piwi-MatKD \#1 F1 embryos. (C) piwi-MatKD \#2 F1 embryos. (D) RT-qPCR for key testis-specific mRNAs in MatKD F1 ovaries, normalized to actin5C RNA levels (left panel). Upper right panel indicates expression level of total phf7 $\left(p h f 7^{T O T}\right)$ and lower right panel 
bioRxiv preprint doi: https://doi.org/10.1101/2021.04.30.442025; this version posted April 30, 2021. The copyright holder for this preprint (which was not certified by peer review) is the author/funder, who has granted bioRxiv a license to display the preprint in perpetuity. It is made available under aCC-BY-NC 4.0 International license.

indicates $p h f 7^{R C}$ relative to $p h f 7^{T O T}$, after each was normalized to actin5C. Two-way ANOVA and Dunnett's Multiple Comparisons Test, ${ }^{*} p<0.05,{ }^{* *} p<0.01,{ }^{* * *} p<0.001$. 
bioRxiv preprint doi: https://doi.org/10.1101/2021.04.30.442025; this version posted April 30, 2021. The copyright holder for this preprint (which was not certified by peer review) is the author/funder, who has granted bioRxiv a license to display the preprint in perpetuity. It is made available under aCC-BY-NC 4.0 International license.

\section{Figure 1}

A

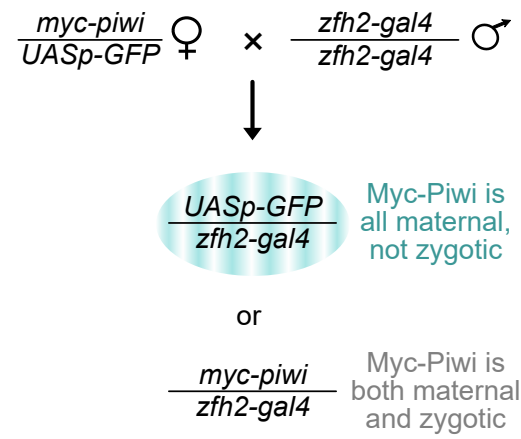

C

Stage 5

PGC Specification
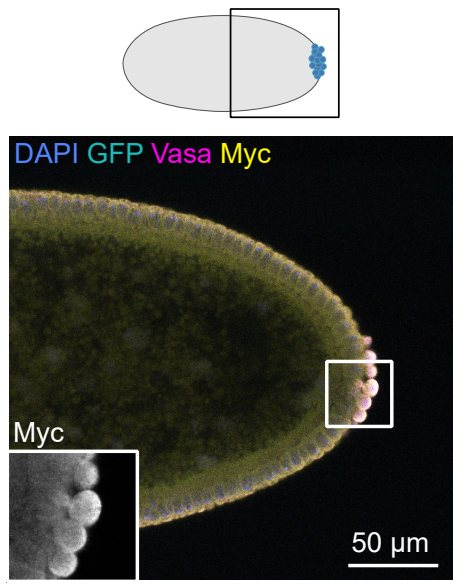

B

$$
\begin{aligned}
& \text { Maternal } \\
& \text { Genotype: } \\
& \text { Zygotic } \\
& \text { Genotype: }
\end{aligned}
$$
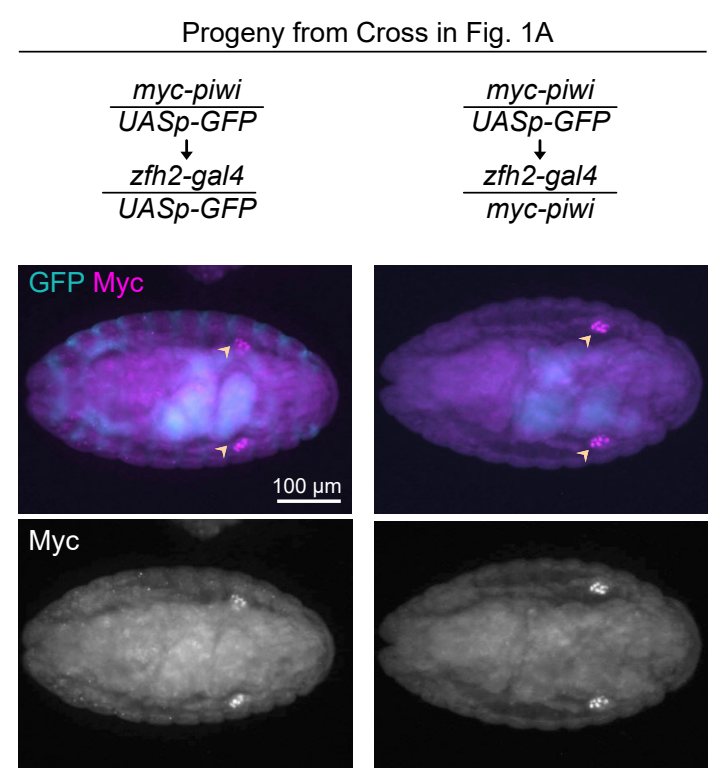

Stage 10 PGC Migration
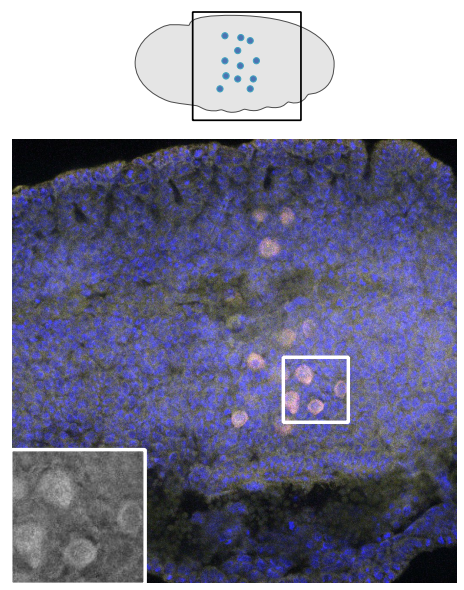

Stage 14 Gonad Coalescence
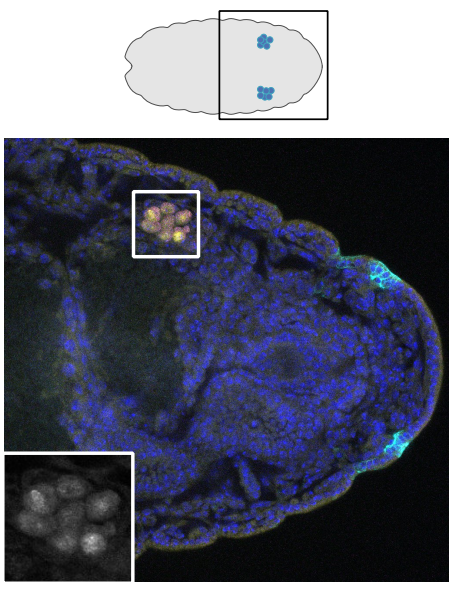

Negative Control

$\frac{U A S p-G F P}{U A S p-G F P}$

$\downarrow$

zfh2-gal4

UASp-GFP

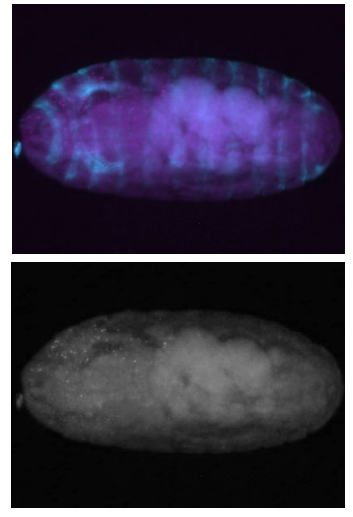

Stage 17

Gonad Coalescence
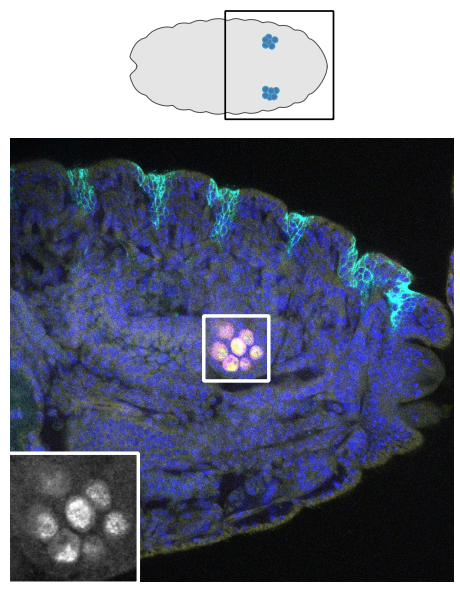
bioRxiv preprint doi: https://doi.org/10.1101/2021.04.30.442025; this version posted April 30, 2021. The copyright holder for this preprint (which was not certified by peer review) is the author/funder, who has granted bioRxiv a license to display the preprint in perpetuity. It is made available under aCC-BY-NC 4.0 International license.

\section{Figure 2}

A

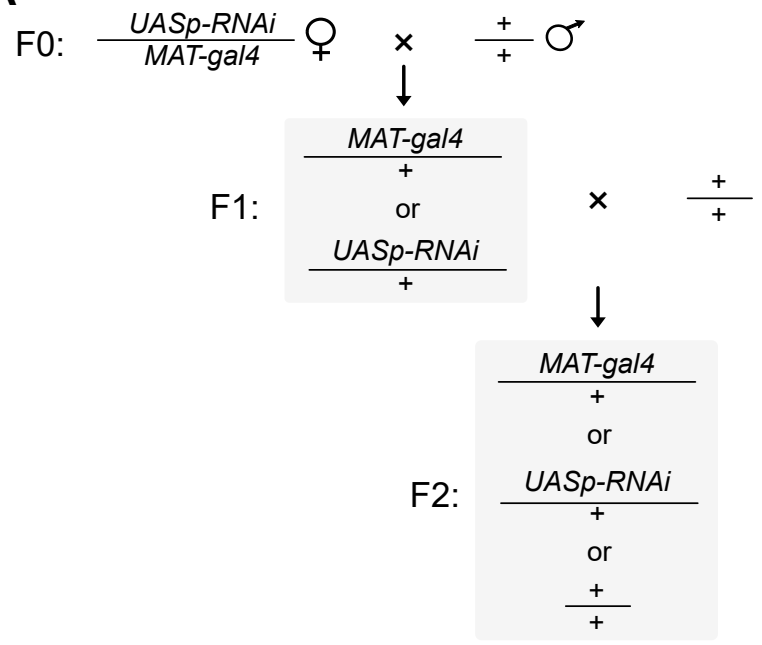

C

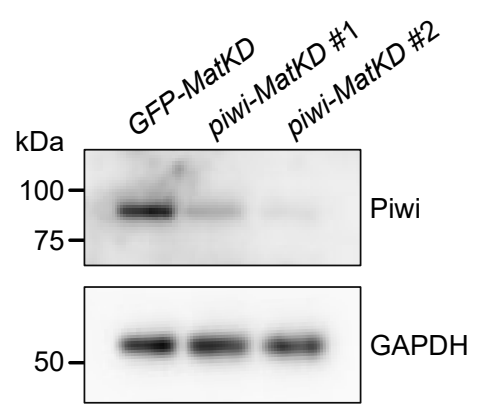

E F1 Females
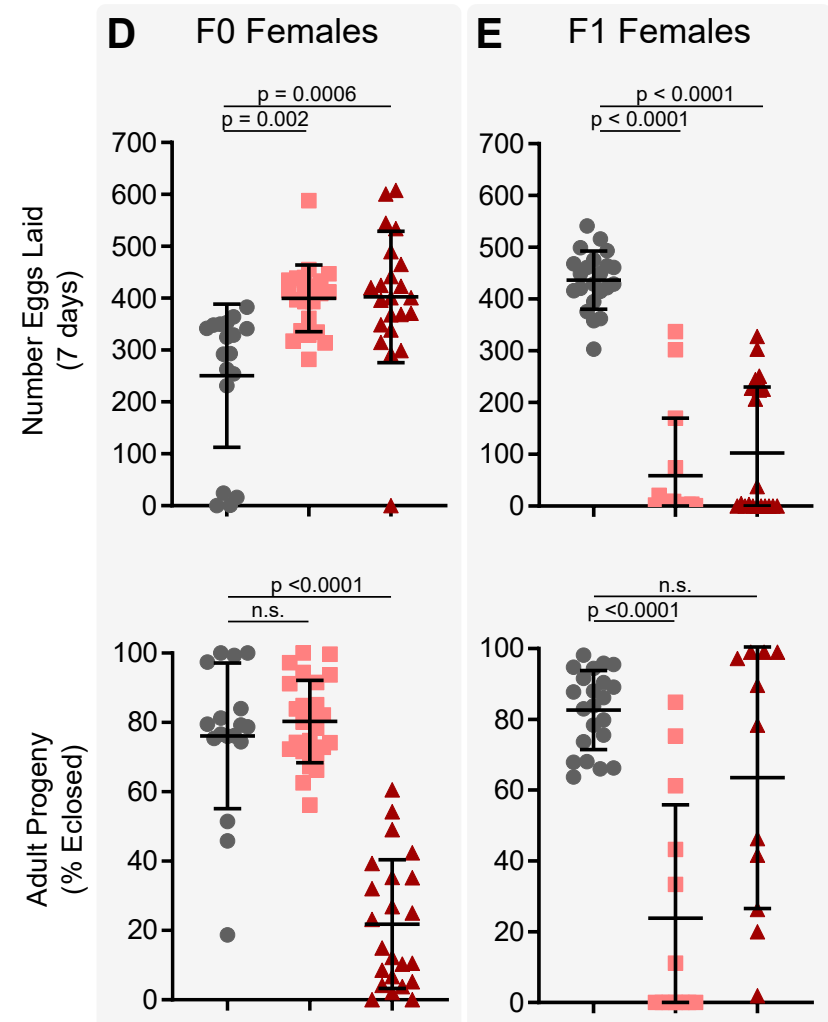

B

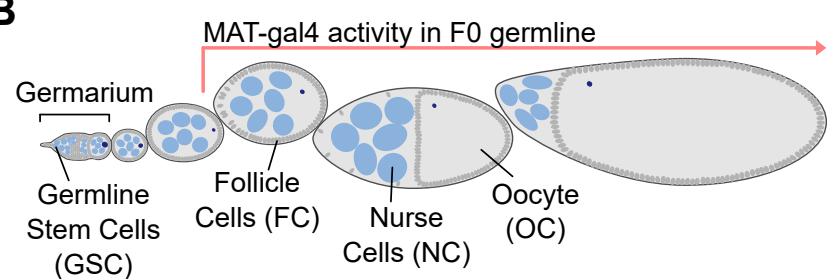

(GSC)
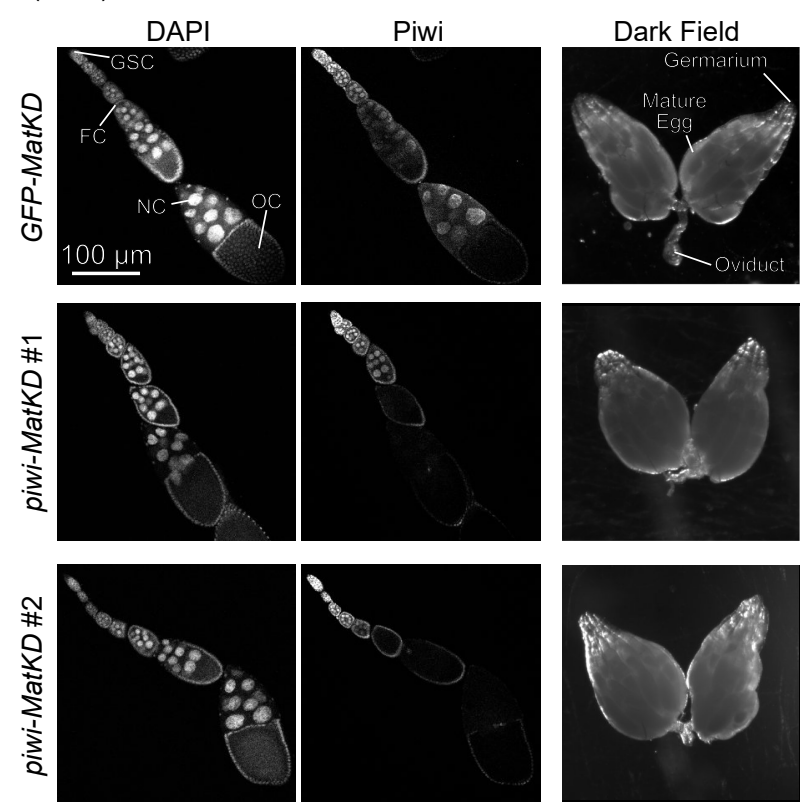

\section{F $\quad$ F1 Males}
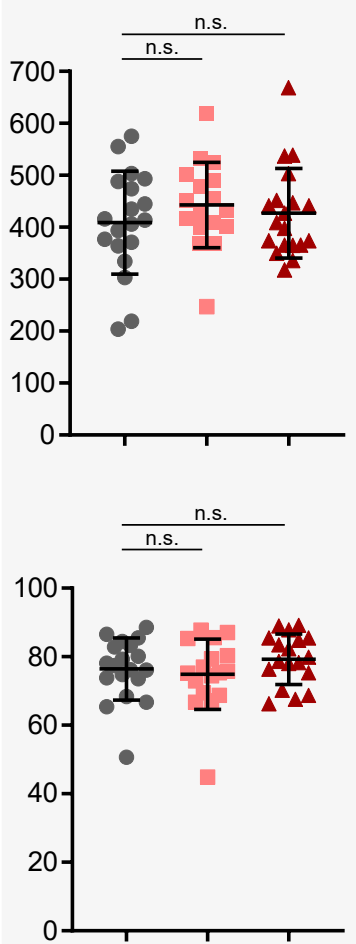

G F2 Females
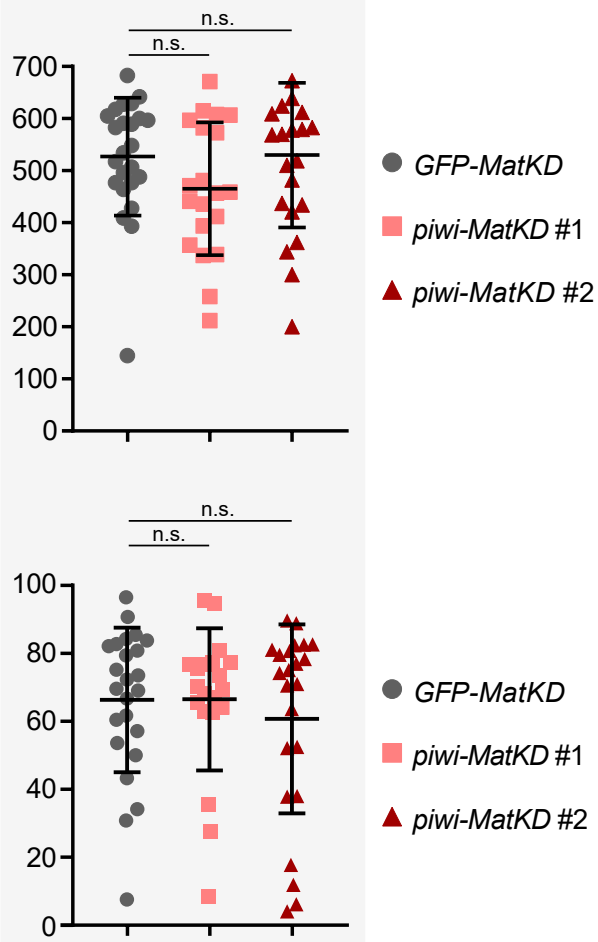
bioRxiv preprint doi: https://doi.org/10.1101/2021.04.30.442025; this version posted April 30, 2021. The copyright holder for this preprint (which was not certified by peer review) is the author/funder, who has granted bioRxiv a license to display the preprint in perpetuity. It is made available under aCC-BY-NC 4.0 International license.

\section{Figure 3}
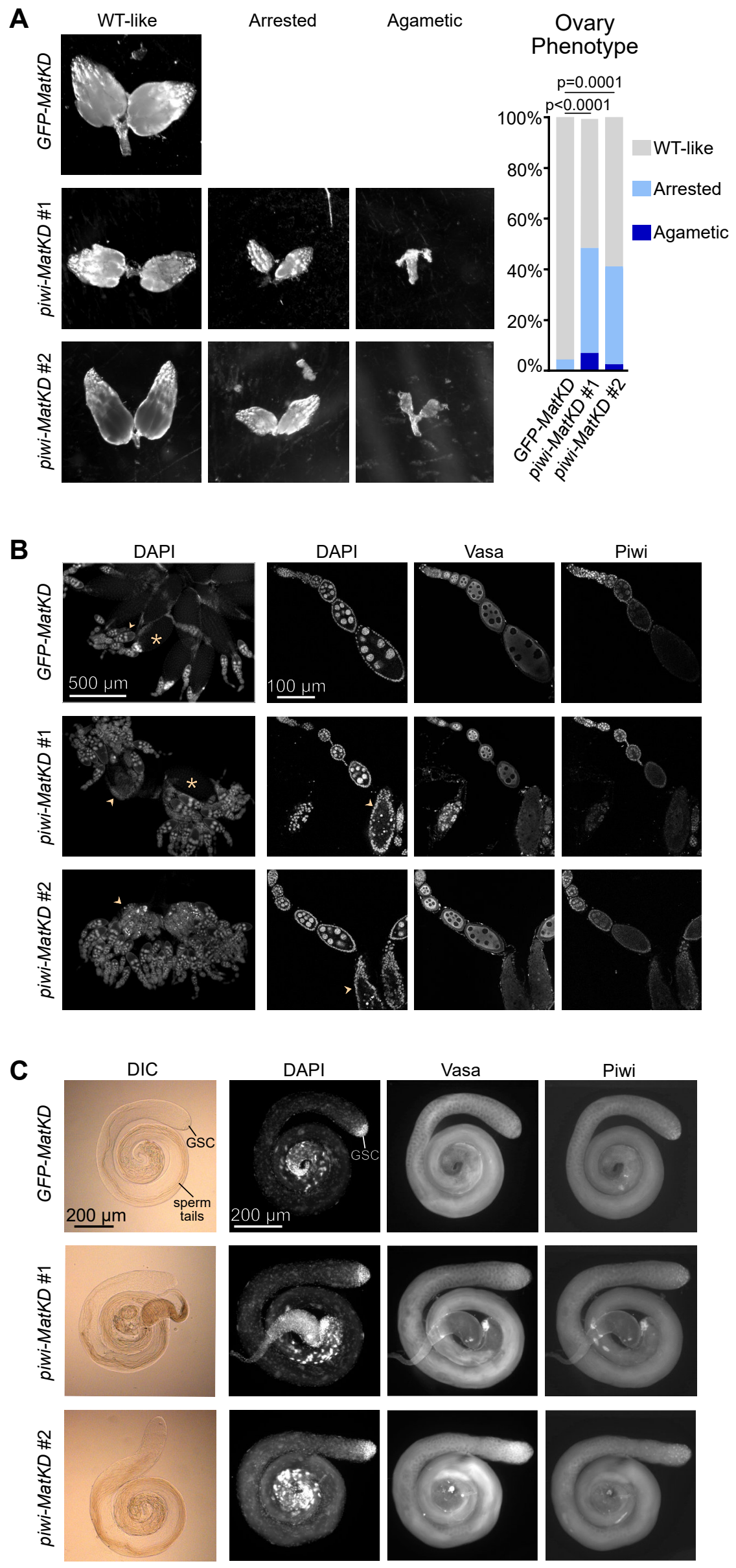
bioRxiv preprint doi: https://doi.org/10.1101/2021.04.30.442025; this version posted April 30, 2021. The copyright holder for this preprint (which was not certified by peer review) is the author/funder, who has granted bioRxiv a license to display the preprint in perpetuity. It is made available under aCC-BY-NC 4.0 International license.

\section{Figure 4}

A

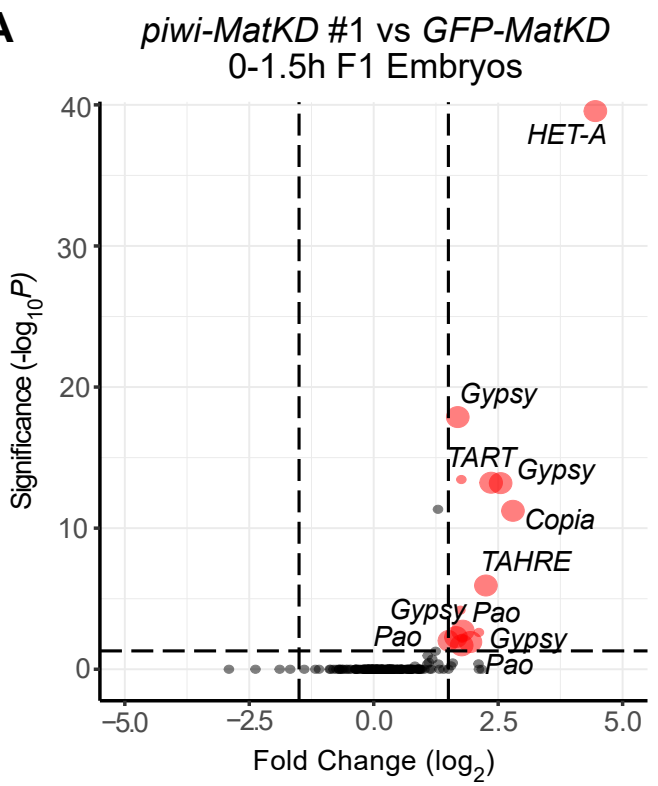

B

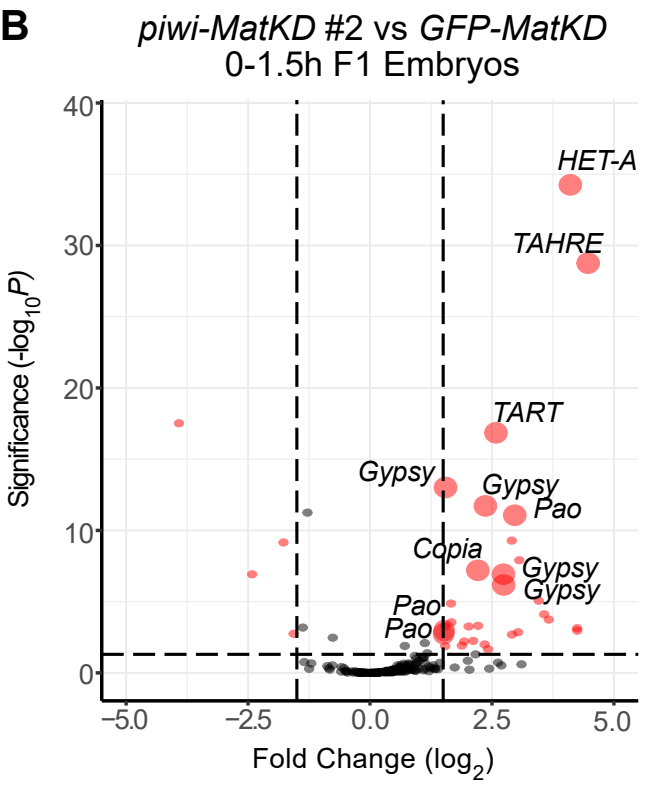

C Transposon Classes

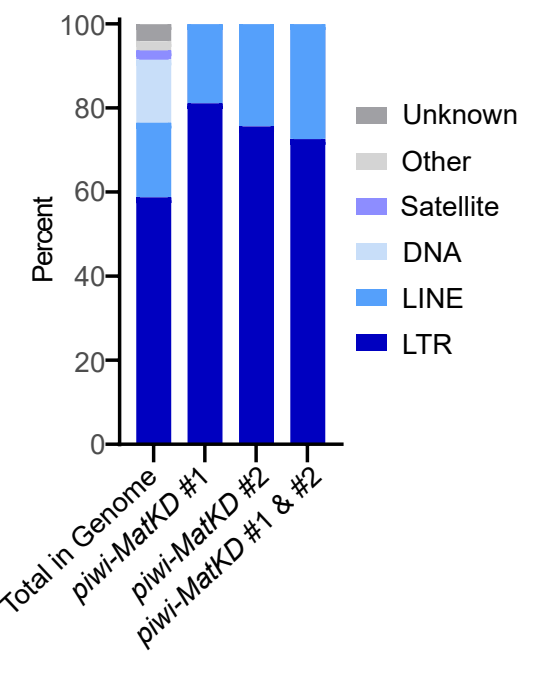

D

Transposon Expression in F1 Ovaries

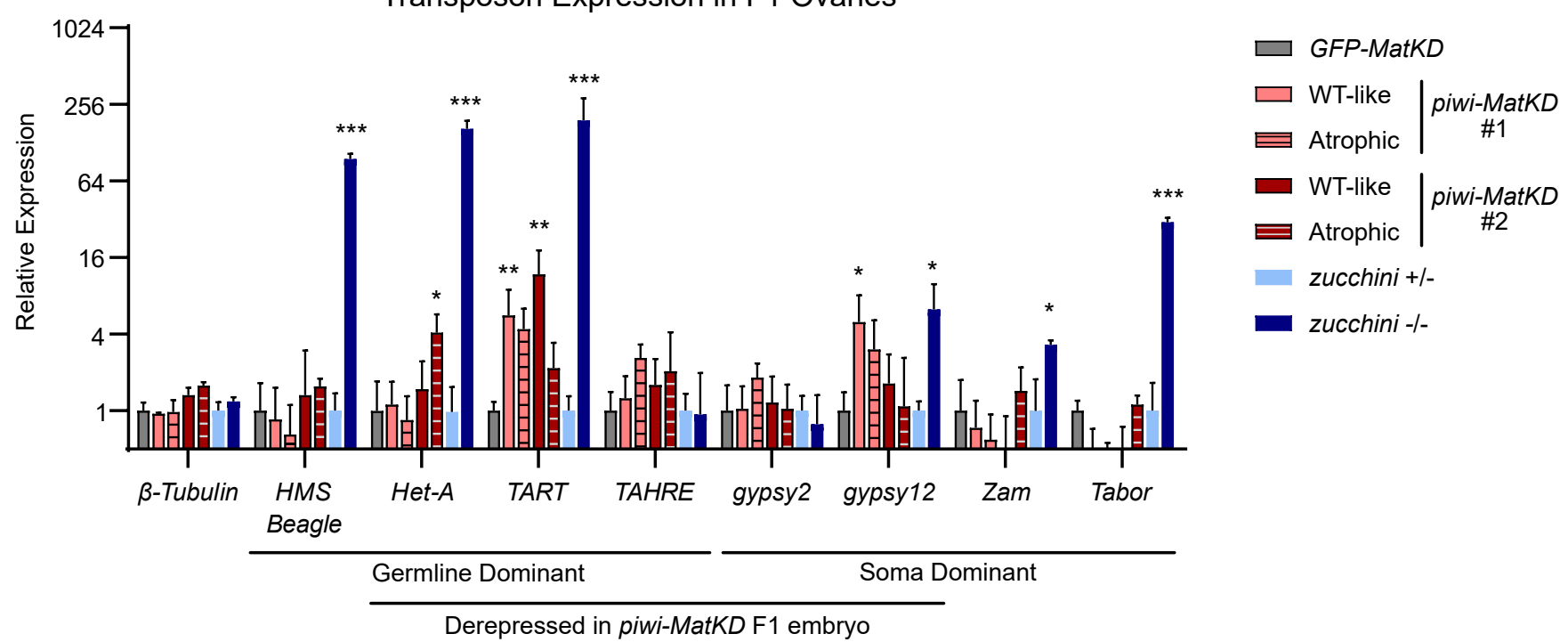


bioRxiv preprint doi: https://doi.org/10.1101/2021.04.30.442025; this version posted April 30, 2021. The copyright holder for this preprint (which was not certified by peer review) is the author/funder, who has granted bioRxiv a license to display the preprint in perpetuity. It is made available under aCC-BY-NC 4.0 International license.

\section{Figure 5}

A piwi-MatKD \#1 vs GFP-MatKD mRNA Expression

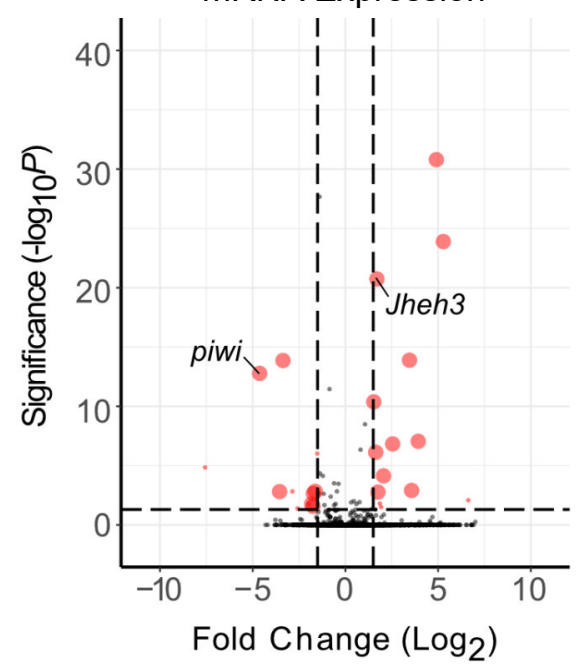

C

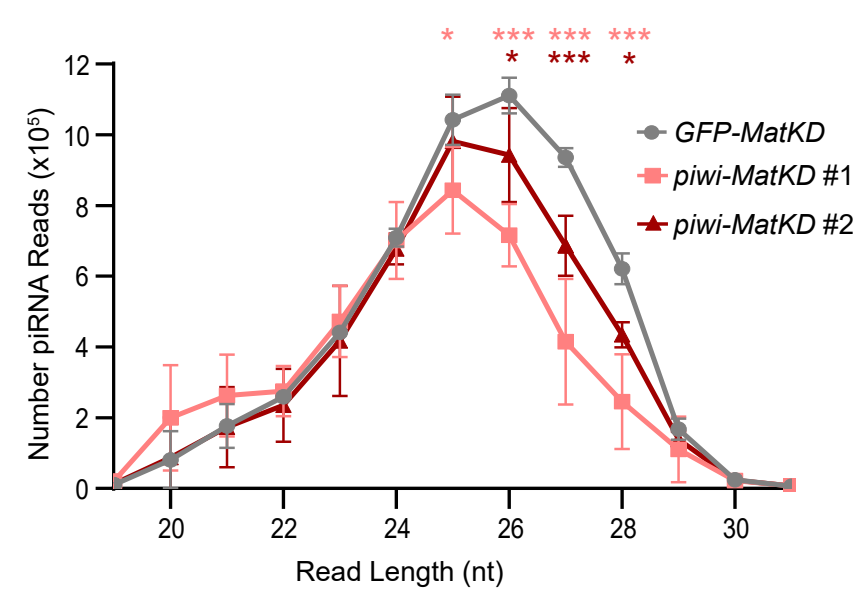

piwi-MatKD \#2 vs GFP-MatKD mRNA Expression

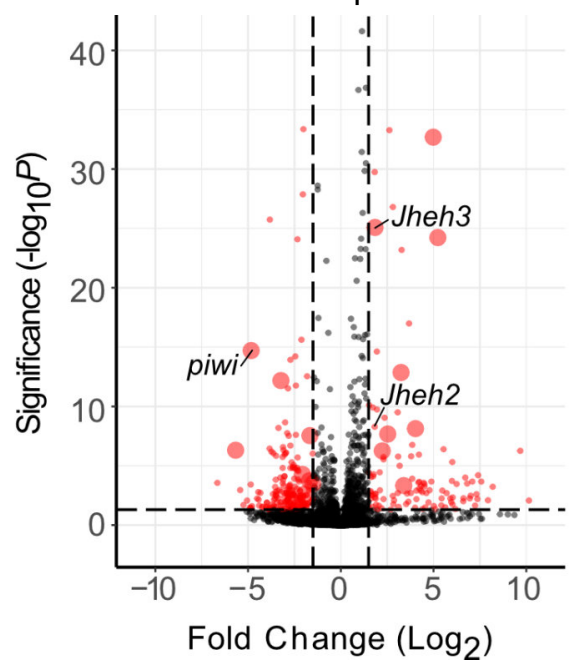

B

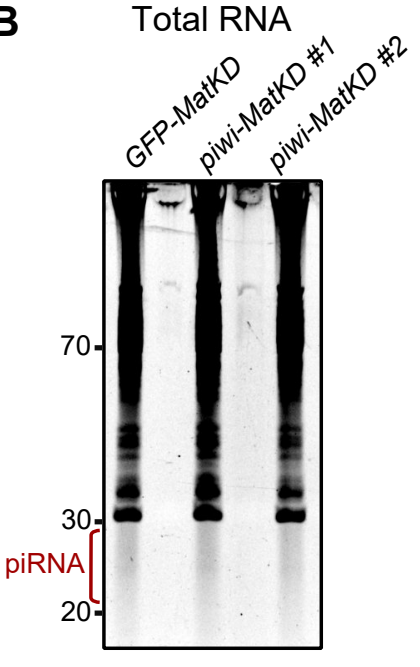

D Nucleotide Identity

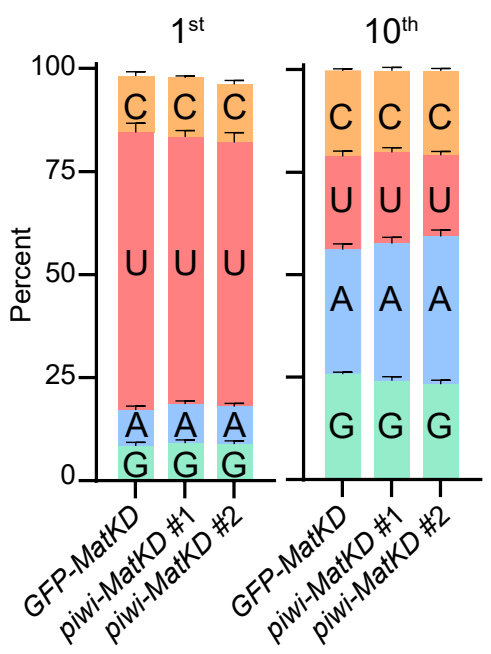

E piRnAs that can target non-TE mRNAs

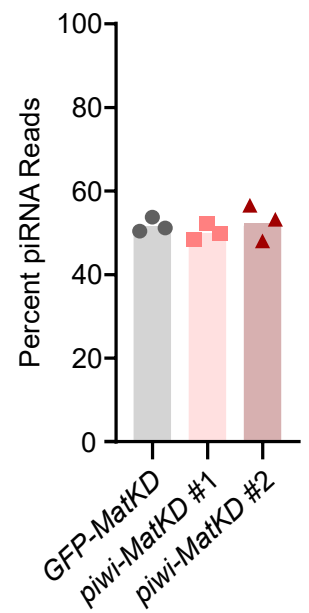

$\mathbf{F}$ piwi-MatKD \#1 vs GFP-MatKD mRNA Targetability by piRNAs

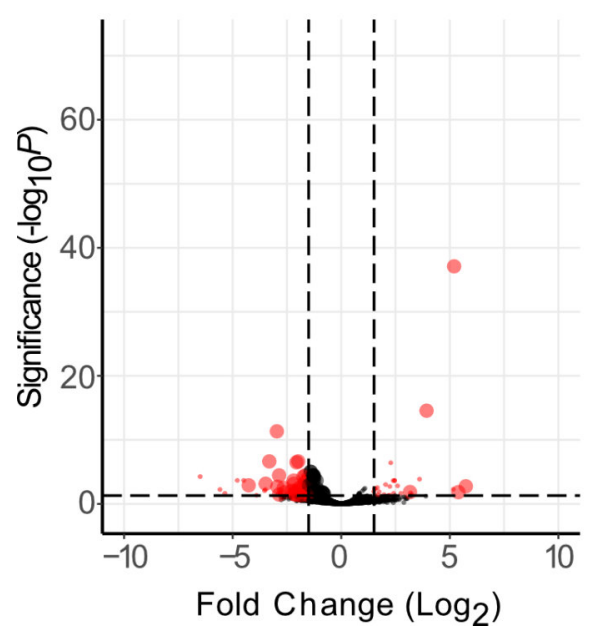

piwi-MatKD \#2 vs GFP-MatKD mRNA Targetability by piRNAs

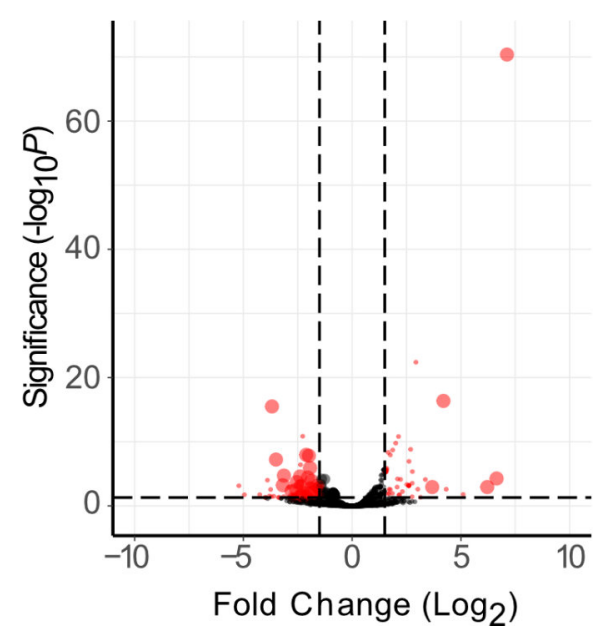

G Antisense piRnAs
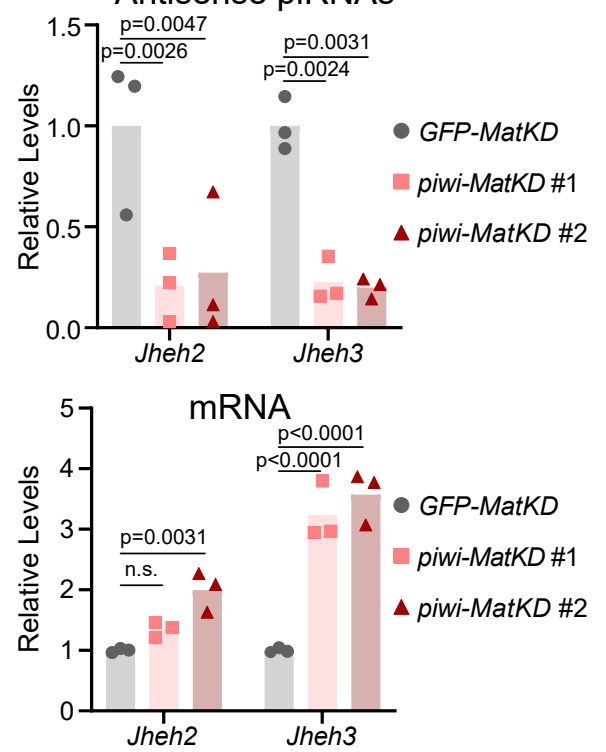
bioRxiv preprint doi: https://doi.org/10.1101/2021.04.30.442025; this version posted April 30, 2021. The copyright holder for this preprint (which was not certified by peer review) is the author/funder, who has granted bioRxiv a license to display the preprint in perpetuity. It is made

Figure 6 available under aCC-BY-NC 4.0 International license.

A
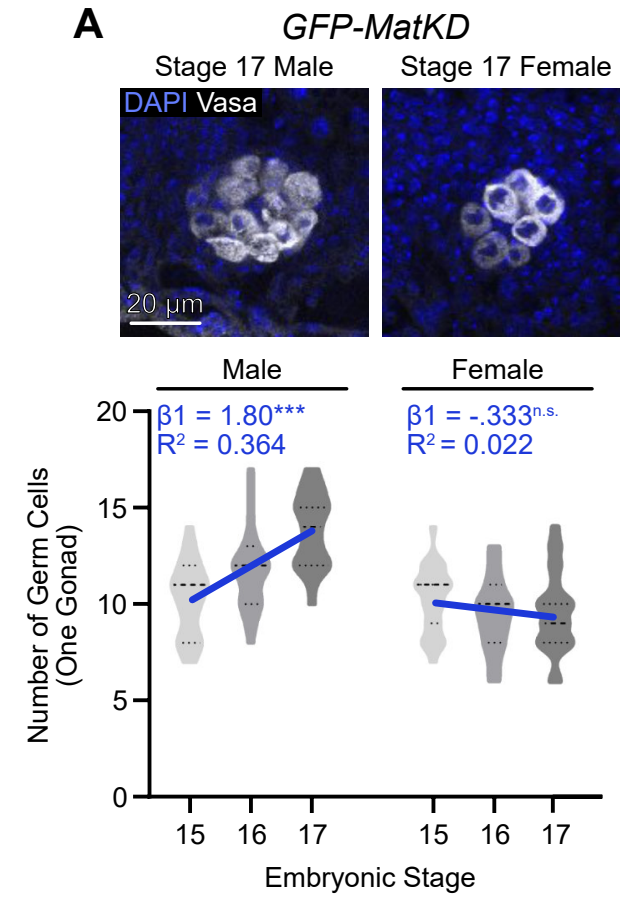

B

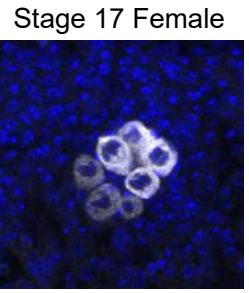

\begin{tabular}{c} 
Female \\
\hline$\beta 1=-.333^{\text {n.s. }}$ \\
$R^{2}=0.022$
\end{tabular}

Embryonic Stage

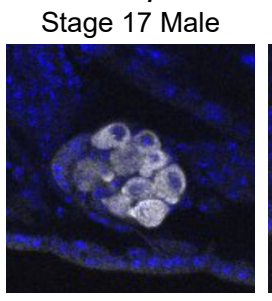

$20-1$

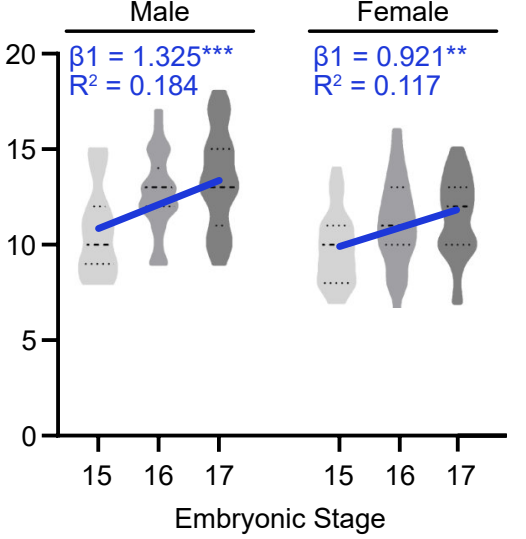

C

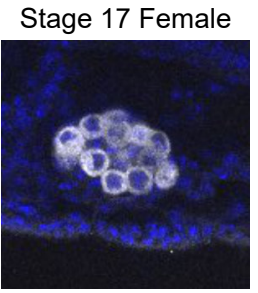

Female

$\beta 1=0.921^{*}$

Embryonic Stage
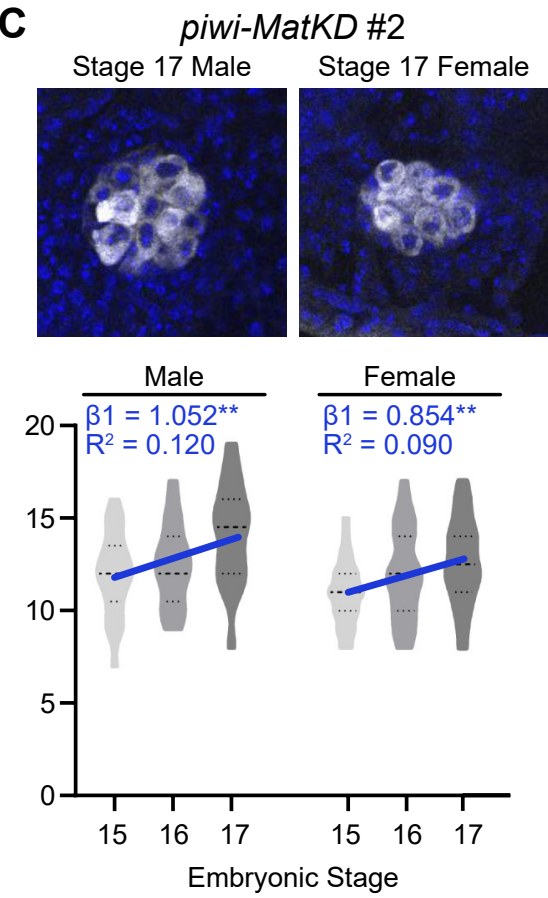

D
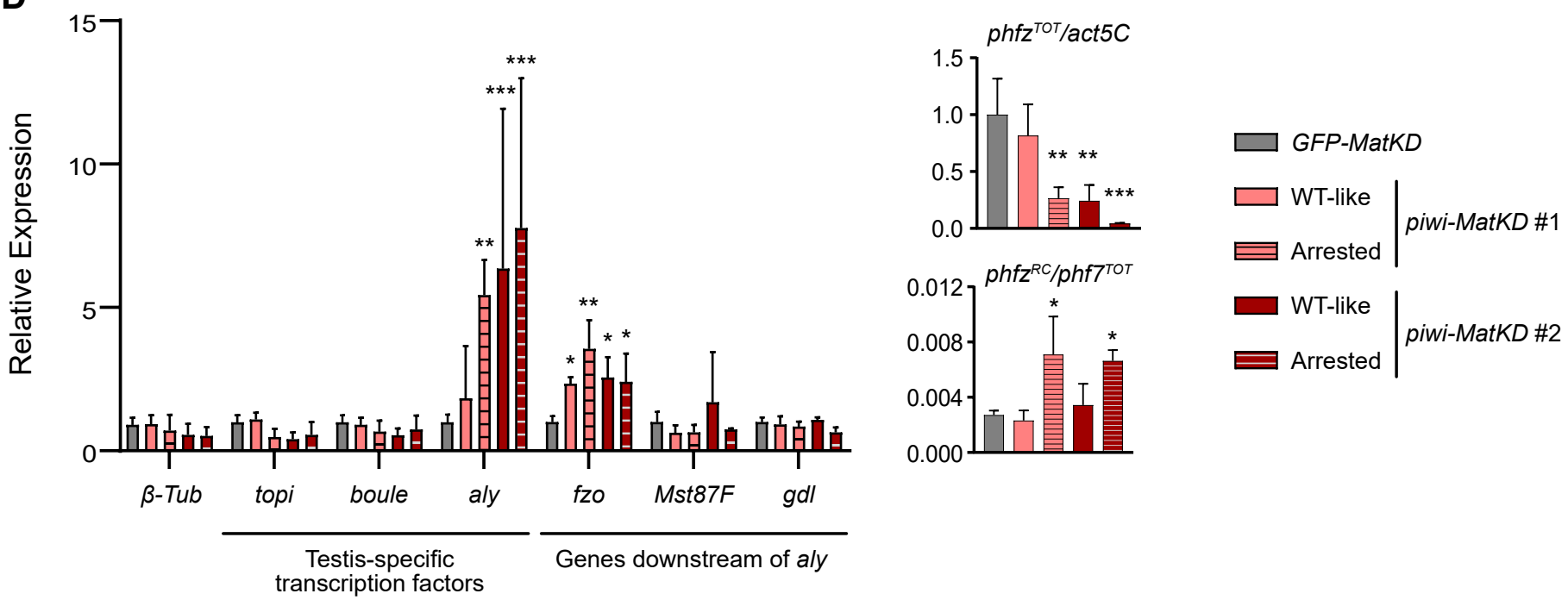